DIVISION OF THE HUMANITIES AND SOCIAL SCIENCES

CALIFORNIA INSTITUTE OF TECHNOLOGY

PASADENA, CALIFORNIA 91125

A CONTRACTION PRINCIPLE FOR FINITE GLOBAL GAMES

Laurent Mathevet

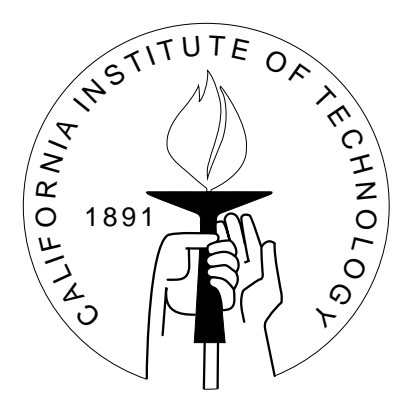

SOCIAL SCIENCE WORKING PAPER 1243R

December 2005

Revised March 2006 


\title{
A Contraction Principle for Finite Global Games
}

\author{
Laurent Mathevet
}

\begin{abstract}
I provide a new proof of uniqueness of equilibrium in a wide class of global games. I show that the joint best-response in these games is a contraction. The uniqueness result then follows as a corollary of the contraction property. Furthermore, the contraction mapping approach provides a revealing intuition for why uniqueness arises: Complementarities in games generate multiplicity of equilibria, but the global games structure dampens complementarities so that only one equilibrium exists. I apply my result to show that uniqueness is obtained through contraction in currency crises and Diamond's search models.
\end{abstract}

JEL classification numbers: C72, D82

Key words: Global games, equilibrium uniqueness, contraction mapping, strategic complementarities, supermodular games 


\title{
A Contraction Principle for Finite Global Games*
}

\author{
Laurent Mathevet
}

\section{Introduction}

Many economic situations present complementarities. If we think of strategies as real numbers, games with strategic complementarities (GSC) are games in which the marginal utility that an agent receives from playing a greater strategy, increases as other agents also play greater strategies. Beyond their intuitive interpretation, these games possess some nice properties in terms of existence of equilibria, characterization of the equilibrium set and comparative statics. However, complementarities often result in multiple equilibria (Takahashi [20]), which requires a theory of equilibrium selection for GSC to have predictive power. Pioneering work by Carlsson and van Damme ([3], CvD hereafter) and Frankel, Morris and Pauzner ([4], FMP hereafter) has provided such a theory of equilibrium selection with the theory of Global Games. In global games, a unique profile survives iterative elimination of dominated strategies. Although global games are widely used in economics (Debt pricing (Morris and Shin [14]), currency crises (Morris and Shin [13]), bank runs (Goldstein and Pauzner [5]), merger waves (Toxvaerd [22])), the proofs of uniqueness by CvD and FMP are intricate, and have left us with a limited understanding of the uniqueness result. My main contribution is to introduce contraction principles into global games to provide a more instructive and intuitively appealing proof of uniqueness.

My result takes a stronger or a weaker form, which depends on the assumptions placed on beliefs. Global games are games with incomplete information and players receive a noisy signal about a random payoff parameter. Conditional on their signal, players formulate beliefs about their opponents' signals. The stronger claim applies when these beliefs are well-behaved or when they satisfy a translation criterion discussed later. Consider the metric space $(\mathcal{C}, d)$ where $\mathcal{C}$ is the set of strategy profiles and $d$ is a metric. Let $b r$ be the (joint) best-response in the global game. I prove the following contraction property

*This paper is a shortened version of the first chapter of my dissertation at Caltech. I am profoundly grateful to my advisors, Federico Echenique and Matt Jackson, for their help and encouragement. I also wish to thank Kim Border, Chris Chambers, Jon Eguia, Chryssi Giannitsarou, Preston McAfee, Andrea Mattozzi, Stephen Morris, Laura Panattoni, Flavio Toxvaerd, and David Young for helpful comments and suggestions. The Division of Humanities and Social Sciences at Caltech and Matt Jackson are gratefully acknowledged for financial support. 
(CP): $d\left(b r(c), b r\left(c^{\prime}\right)\right)<d\left(c, c^{\prime}\right)$ for all $\left(c, c^{\prime}\right) \in \mathcal{C}^{2}$ with $c \neq c^{\prime}$. Since global games are GSC, Tarski's theorem applies to the best-response to show the existence of a fixed point (See Topkis [21]). If the best-response had two fixed points, then (CP) would lead to a contradiction. Thus the equilibrium must be unique.

The weaker claim applies when there is no requirement on beliefs. Players receive noisy signals but the precision of their signal increases as some noise parameter $\nu$ vanishes. Parameterize the global game by its noise level and let $\operatorname{br}(\nu)$ be the joint bestresponse in this game. Define $\mathcal{C}_{\nu}$ as the set of pairs $\left(c, c^{\prime}\right)$ for which $\operatorname{br}\left(\nu^{\prime}\right)$ satisfies $(\mathbf{C P})$ for all $\nu^{\prime}<\nu$. Then, I prove that $\cup_{\nu>0} \mathcal{C}_{\nu}=\mathcal{C}^{2}$. In words, the best-reply is a contraction in the limit because the set on which it contracts approaches the whole set as $\nu$ goes to zero. For every noise level, the global game is a GSC and thus an equilibrium exists. The contraction property then implies that the equilibrium must be unique in the limit as the noise disappears.

This contraction result provides a formal basis for the existing intuition that the global games structure lessens complementarities to the point where we obtain a unique equilibrium. GSC have a coordination-game "flavor" that leads to multiple equilibria; this relationship can be traced to how strong complementarities are. But, global games are GSC and there is a unique equilibrium in the limit. Therefore, the complementarities in global games must somehow be lessened as the noise level shrinks. This intuition is set out in Vives [25], who establishes that complementarities are moderated in a specific example of a global game. My paper thereby provides the first result that formalizes how the global games structure dampens complementarities in a general global game framework. The positive slope of the best-reply is a measurement of the strength of complementarities; I show that the best-reply is a contraction, hence it is not "too increasing." Therefore the complementarities cannot be too strong which leads to uniqueness.

My results require two assumptions in addition to FMP's, but these assumptions are either automatically satisfied or unnecessary in $2 \times 2$ games. ${ }^{1}$ With some qualifications, the contraction result is, for $2 \times 2$ games, as general as the previous results of uniqueness in the literature. Those games are of interest because it is the class of games analyzed in the seminal work by CvD. These assumptions come into play in general finite games, but the structure is still general enough to allow for applications to well-known models like currency crises and Diamond's search. ${ }^{2}$ In the currency crises model, one of these assumptions is trivially satisfied, and the other one is a natural property of the exchange rate. In the Diamond's search model, both assumptions are satisfied in the traditional setting with convex cost functions.

Moreover, the contraction mapping principle sharply leads to uniqueness; once it is established, uniqueness is transparent. FMP's uniqueness result is a significant generalization of the infection arguments of $\mathrm{CvD}$, and it still relies on iterated strict dominance. With the contraction mapping, solving for iterated elimination of dominated strategies is obviated and FMP's (and thus CvD's) uniqueness result becomes a corollary of the

\footnotetext{
${ }^{1}$ The two additional assumptions are made on payoffs. One is trivially satisfied in $2 \times 2$ games and the other one turns out to be unnecessary.

${ }^{2}$ There is also an application of the contraction result to an arms race model that can be found in Mathevet [10].
} 
contraction argument.

In addition, I argue in Section 2 that the contraction result is simpler to establish than the results in previous literature. The intuition for the proof of the contraction in the $2 \times 2$ case, which is presented in Section 4.1.1, is straightforward and easily carries over to the general case. However, the notation is somewhat heavy. Section 5 gives the intuition for this generalization.

Finally, FMP's result relies on a two-step argument where uniqueness in the actual bayesian game is shown from a simplified version of the same game. Uniqueness follows by continuity to this easier environment where uniqueness is more easily verified. This methodology is not fully informative about the underlying mechanisms of the general case. While I also consider both versions of the game, I prove uniqueness separately. One of the advantages of treating the general game directly is that, unlike FMP, I show there is not always need for a vanishing noise to get uniqueness.

We now discuss some of the related literature. Mason and Valentinyi [9] used a contraction mapping approach to establish uniqueness of equilibrium in a class of incomplete information games. Their argument requires sufficiently large perturbations from the complete information case and that players signals be sufficiently independent, or uninformative about others'. Their theory mainly imposes structure on beliefs. On the other hand, my paper studies global games, and so the uniqueness typically arises from very small perturbations from the complete information game. In global games, players' signals become fully informative and correlated as the noise level goes to zero. My uniqueness result instead imposes structure on payoffs. Finally, in a recent paper, Oury [18] extended the uniqueness result of FMP to multidimensional global games. It is an important step forward in global games, but her argument is a generalization of FMP's proof technique which is different from my contraction approach.

\section{A Motivating Example}

Consider this example taken from CvD. Two players are deciding whether to invest. Each player receives a net profit that depends not only on her action and her opponent's action, but also on a fundamental of the economy, denoted by $\theta \in \mathbb{R}$. The payoff matrix is the following:

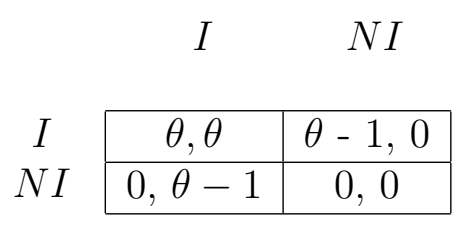

Notice that $N I$ is strictly dominant for values of $\theta$ strictly below $\underline{\theta}=0$, and $I$ is strictly dominant for values of $\theta$ strictly above $\bar{\theta}=1$. Further, there are two strict Nash equilibria when $\theta \in(0,1)$. This is the multiple-equilibrium problem described earlier. Which one should be played? 
We would like to have a paradigm to select one of these equilibria for each value of $\theta \in(0,1)$. I show how introducing incomplete information solves the problem because the best-response is a contraction and hence gives uniqueness in the incomplete information version of the game. I shall then use CvD and FMP's techniques in this example to compare their approach to mine.

The incomplete information arises when payoffs are disturbed. State $\theta$ is drawn from a continuous distribution $\phi$ whose support is the real line. Each player observes a noisy signal $s_{i}=\theta+\nu \epsilon_{i}$ of the fundamental, where $\epsilon_{i}$ is distributed according to some cdf $F_{i}\left[-\frac{1}{2}, \frac{1}{2}\right]$, and $\nu>0$. The information structure $\left(\phi,\left\{F_{i}\right\}\right)$ allows each player $i$ (through Bayes' rule) to construct a distribution function $\Omega_{i}\left(s_{j} \mid s_{i}, \nu\right)$, or simply $\Omega_{i}\left(s_{i} \mid s_{j}\right)$, representing her beliefs about $j$ 's signal upon receiving $s_{i}$. Let us assume beliefs are translation increasing for all $i$ which I define next. The cdf $\Omega_{i}$ is translation increasing if for all $s_{j}$ and $s_{i}, \Omega_{i}\left(s_{j}+\Delta \mid s_{i}+\Delta^{*}\right) \leq \Omega_{i}\left(s_{j} \mid s_{i}\right)$ whenever $\Delta^{*}$ and $\Delta$ are such that $\Delta^{*} \geq \Delta$. It means that $i$ believes that $j$ 's signal is more likely to be below $s_{j}$ when receiving $s_{i}$ than it is below $s_{j}+\Delta$ when receiving $s_{i}+\Delta^{*}$.

In the incomplete information game, it turns out that we only need to consider monotone strategies (See Section 3.3). So, a strategy for $j$ is fully defined by a cutoff $c_{j} \in[0,1]$ :

$$
a_{j}\left(s_{j}\right)= \begin{cases}N I & \text { if } \quad s_{j}<c_{j} \\ I & \text { if } \quad s_{j} \geq c_{j}\end{cases}
$$

Denote by $b r_{i}\left(c_{j}\right)$ that cutoff between actions $N I$ and $I$ that corresponds to $i$ 's bestresponse to $c_{j}$. I shall prove that for any $i \in N, b r_{i}$ is a contraction. That is, $\mid b r_{i}\left(c_{j}^{\prime}\right)-$ $b r_{i}\left(c_{j}\right)|<| c_{j}^{\prime}-c_{j} \mid$ for all $c_{j}^{\prime}, c_{j} \in[0,1]$ such that $c_{j}^{\prime} \neq c_{j}$. Formally, $b r_{i}\left(c_{j}\right) \equiv c_{i}$ and if signals enter payoff directly ${ }^{3}$ then signal $c_{i}$ is such that:

$$
\left(c_{i}-1\right) \Omega_{i}\left(c_{j} \mid c_{i}\right)+c_{i}\left(1-\Omega_{i}\left(c_{j} \mid c_{i}\right)\right)=0
$$

or equivalently,

$$
\Omega_{i}\left(c_{j} \mid c_{i}\right)=c_{i} \equiv b r_{i}\left(c_{j}\right) .
$$

In words, player $i$ 's expected payoff to playing 1 is bigger than her expected payoff to playing 0 if and only if the signal exceeds $c_{i}$. Therefore, cutoff strategy $b r_{i}\left(c_{j}\right)$ is the best-reply to $c_{j}$.

From (1), it is straightforward to show the contraction property using translation increasing beliefs. Take any $\Delta>0, c_{j} \in[0,1]$ and consider an increase from $c_{j}$ to $c_{j}+\Delta$. By means of contradiction, suppose $b r_{i}\left(c_{j}+\Delta\right)-b r_{i}\left(c_{j}\right)=\Delta^{*} \geq\left(c_{j}+\Delta\right)-c_{j}=\Delta$. Since $c_{i} \equiv b r_{i}\left(c_{j}\right)$, then $c_{i}+\Delta^{*} \equiv b r_{i}\left(c_{j}+\Delta\right)$. Thus, by (1), $c_{i}=\Omega_{i}\left(c_{j} \mid c_{i}\right)$ and $c_{i}+\Delta^{*}=\Omega_{i}\left(c_{j}+\Delta \mid c_{i}+\Delta^{*}\right)$. But then translation increasing beliefs would imply $c_{i}+\Delta^{*} \leq c_{i}$, which is a contradiction because $\Delta^{*}>0$. Therefore, $b r_{i}\left(c_{j}+\Delta\right)-b r_{i}\left(c_{j}\right)<\Delta$. Moreover, $b r_{i}$ is monotone increasing and so we have $b r_{i}\left(c_{j}\right)-b r_{i}\left(c_{j}+\Delta\right)<\Delta$. As $c_{j}$, $\Delta$ and $\Delta^{*}$ were arbitrary, we conclude $\left|b r_{i}\left(c_{j}^{\prime}\right)-b r_{i}\left(c_{j}\right)\right|<\left|c_{j}^{\prime}-c_{j}\right|$ for all distinct $c_{j}$ and $c_{j}^{\prime}$.

\footnotetext{
${ }^{3}$ This assumption of "private values" is the simplification of the actual bayesian game that I alluded to in the introduction. This easier environment is enough to make the point for the contraction result. See Section 3.2 for a discussion.
} 
Notice that contraction of the joint best-response $b r=\times_{i \in N} b r_{i}$ follows immediately from the above. Take any $c=\left(c_{i}, c_{j}\right), c^{\prime}=\left(c_{i}^{\prime}, c_{j}^{\prime}\right)$ in $[0,1]^{2}$ and let $d\left(c, c^{\prime}\right)=\max _{k \in N}\left|c_{k}^{\prime}-c_{k}\right|$. Then $d\left(\operatorname{br}(c), \operatorname{br}\left(c^{\prime}\right)\right)<d\left(c, c^{\prime}\right)$. This definition of a contraction is not the usual one. Technically, $b r$ is a pseudo-contraction or a shrinking map.

To complete the proof of uniqueness, recall that global games are GSC and so by Tarski's fixed point theorem an equilibrium exists. ${ }^{4}$ The contraction property implies uniqueness. ${ }^{5}$

In the above example, translation increasing beliefs have a nice interpretation. Intuitively, it means that given two players $i$ and $j$, player $i$ believes player $j$ is more likely to play her lower action whenever player $i$ increases her strategy (lowers her cutoff) more than player $j$. By the complementarities, such a big increase cannot be optimal. Therefore, this property of the beliefs prevents strategies from getting carried away by the complementarities by tempering their effect.

The translation property drives the result but how restrictive is it? Many distributions are translation increasing like normal, double exponential distributions centered at $s$, and it is a common feature of location-scale families. We shall see that it is also satisfied whenever $\Omega_{i}$ is derived from the combination of a uniform prior and any bounded noise function. Furthermore, Lemma 2 in Section 7 shows that even if we do not make this assumption, it holds in the limit as $\nu$ goes to zero. This Lemma demonstrates that for any prior the global game information structure naturally tends to be increasing in translation for small noise, which is why uniqueness is then reached in the limit only.

Equilibrium selection in the complete-information version of the game is obtained via uniqueness in its incomplete-information version. For all $i, s_{i}=\theta+\nu \epsilon_{i}$ and so $s_{i}$ gets closer to $\theta$ as $\nu$ becomes small because $\epsilon_{i} \in[-1 / 2,1 / 2]$. Therefore, for a.e realization of the signals in the unique equilibrium, players play arbitrarily close to some Nash equilibrium of the complete information game as $\nu$ goes to zero.

Now I turn to the alternative techniques that have been proposed so far and I use them to prove uniqueness in the investment game. ${ }^{6}$

Like FMP, suppose the prior is uniform and use iterated dominance. The largest strategy that rationally can be played by player $j$ because of the dominance regions is $c^{j}=0$. In this case, $b r_{i}(0)=l_{1} \geq 0$. The least strategy that rationally can be played by player $j$ is $c^{j}=1$, to which $i$ best-responds by playing $b r_{i}(1)=u_{1}$ which is less than 1 . Given the strategies that player $j$ knows she can induce for player $i$, only her strategies with cutoff in $U_{2} \subset\left[l_{1}, u_{1}\right]$ are not strictly dominated. We iterate on this logic ad infinitum. By Milgrom-Roberts [11], iterated dominance leads to the existence of a

\footnotetext{
${ }^{4}$ The contraction property alone does not ensure the existence of a fixed point without further information on its domain (See Remarks in Section 4.1.2). However, this existence problem is made vacuous by the fact that global games are GSC, and an equilibrium always exists in GSC.

${ }^{5}$ Suppose the joint best-response $b r$ has at least two fixed points, $c$ and $c^{\prime}$. Then by the contraction property: $d\left(c, c^{\prime}\right)=d\left(b r(c), b r\left(c^{\prime}\right)\right)<d\left(c, c^{\prime}\right)$, a contradiction.

${ }^{6}$ What follows is the application of Section "Intuition for Limit Uniqueness" from FMP [4].
} 
largest and a least equilibrium in supermodular games: $\bar{a}^{*}$ and $\underline{a}^{*}$.

From there, FMP used a proof by contradiction which relies on a translation argument. By way of contradiction, say $\bar{a}^{*} \neq \underline{a}^{*}$ and in particular, the corresponding equilibrium cutoff strategies are defined by $\bar{c}$ and $\underline{c}$ in $[0,1] \cdot{ }^{7}$ Now, translate the smallest surviving strategy $\underline{a}^{*}$ by $\mu>0$, until it lies completely but not strictly above the largest surviving strategy. Then denote one of the tangency points with $\bar{a}^{*}$ by $s^{*}$. Let $\tilde{a}$ be the translated strategy. Since $\bar{a}^{*}$ and $\underline{a}^{*}$ are equilibria, we know that $b r\left(\bar{a}^{*}, s^{*}\right)=b r\left(\underline{a}^{*}, s^{*}+\mu\right)$. By supermodularity of the payoffs in actions: $\operatorname{br}\left(\tilde{a}, s^{*}\right) \geq \operatorname{br}\left(\bar{a}^{*}, s^{*}\right)=1$. Then since the prior is uniform, the action distribution a player expects upon receiving $s^{*}$ when her opponent plays according to the translated strategy is the same as the one she expects upon receiving signal $s^{*}+\mu$, when she thinks her opponent follows $\underline{a}^{*}$. But, the strict complementarities of the payoffs in action and signal implies that when she gets the higher signal of $s^{*}+\mu$, then her best-reply to $\underline{a}^{*}$ is actually strictly greater than her best-reply to $\tilde{a}$ when getting $s^{*}$. This is a contradiction: $(1=) \operatorname{br}\left(\underline{a}^{*}, s^{*}+\mu\right)=\operatorname{br}\left(\bar{a}^{*}, s^{*}\right) \leq \operatorname{br}\left(\tilde{a}, s^{*}\right)<\operatorname{br}\left(\underline{a}^{*}, s^{*}+\mu\right)(=1)$. Therefore, $\mu$ must be zero and so $\bar{a}^{*}=\underline{a}^{*}$.

\section{Game and Assumptions}

I formally define each component of a global game and then I give their interpretation in the model.

A (finite) global game is a collection $(\Gamma(\nu))_{\nu>0}$, where each $\Gamma(\nu)$ is a tuple $\left(N,\left(A_{i}, \succeq_{i}\right.\right.$ )$\left._{N}, \phi,\left(\tau_{i}(., \nu), f_{i}\right)_{N}, g,\left(\pi_{i}\right)_{N}\right)$ with the following meaning. The set of players is $N=$ $\{1, \ldots, n\}$ and, action sets $A_{i}$ are subsets of $\mathcal{A}$ where $\mathcal{A}$ is a vector space. Further, $A_{i}=\left\{a_{i, 1}, \ldots, a_{i, m_{i}}\right\}$ is a chain with the binary relation $\succeq_{i}$ such that $a_{i, 1} \prec_{i} \ldots \prec_{i} a_{i, m_{i}}$. A typical element of the joint action set $\prod_{i} A_{i}$ is $\left(a_{i}, a_{-i}\right)$ for any $i$. The function $\phi$ is a continuous density with convex support on $\mathbb{R}$. The function $\tau_{i}$ is the signaling technology, mapping $\mathbb{R} \times \mathbb{R}_{+} \times\left[-e_{i}, e_{i}\right]$ for some $e_{i}>0$ to $\mathbb{R}$ - a typical element of this product of sets is $\left(\theta, \nu, \epsilon_{i}\right)$ - and it is continuous and strictly increasing in all its arguments. Let $e^{*}=\max \left\{e_{i}: i \in N\right\}$. For each $\left(\nu, \epsilon_{i}\right), \theta \mapsto \tau_{i}\left(\theta, \nu, \epsilon_{i}\right)$ is a homeomorphism between $\mathbb{R}$ and $\mathbb{R}$ and for each $(\theta, \nu), \epsilon_{i} \mapsto \tau_{i}\left(\theta, \nu, \epsilon_{i}\right)$ is a homeomorphism between $\left[-e_{i}, e_{i}\right]$ and $\left[\tau_{i}\left(\theta, \nu,-e_{i}\right), \tau_{i}\left(\theta, \nu, e_{i}\right)\right]$. The inverse of these functions will respectively be denoted $\tau_{\theta}^{-1}$ and $\tau_{\epsilon_{i}}^{-1}$. The function $f_{i}$ is a positive density with support $\left[-e_{i}, e_{i}\right]$. Let $(\mathcal{H}, \succeq)$ be a chain and $g: \prod_{j \neq i} A_{j} \rightarrow \mathcal{H}$ be a continuously increasing surjection that carries action profiles into $\mathcal{H}$. Finally, player $i$ 's payoff is given by the real-valued function $\pi_{i}$ for any $i$. If player $i$ chooses action $a_{i} \in A_{i}$, her payoff is $\pi_{i}\left(a_{i}, g\left(a_{-i}\right), \theta\right)$ where $a_{-i}=\left(a_{j}\right)_{j \neq i}$ denotes the action profile of $i$ 's opponents.

A state $\theta \in \mathbb{R}$ is drawn from the real line according to $\phi$ whose support contains a large interval $[\underline{\theta}, 2 \bar{\theta}-\underline{\theta}]$ defined later. Then each player $i$ observes a signal $s_{i}=\tau_{i}\left(\theta, \nu, \epsilon_{i}\right)$ about $\theta$, where $\nu>0$ is a parameter that determines the degree of noise in $\tau_{i}$. Each $\epsilon_{i}$ is distributed according to $f_{i}$. The signal structure is given by function $\tau_{i}$ which has the property to reveal the state if there is no noise or if $\nu=0$, that is, $\tau_{i}\left(\theta, 0, \epsilon_{i}\right)=$ $\tau_{i}(\theta, \nu, 0)=\theta$. There is a case of particular interest, $\tau_{i}\left(\theta, \nu, \epsilon_{i}\right)=\theta+\nu \epsilon_{i}$ for all $i \in N$.

\footnotetext{
${ }^{7}$ Recall the game is symmetric.
} 
I refer to this case as the linear signaling technology case. The noises are assumed to be conditionally independent of one another and of the state of the world: each $\epsilon_{i}$ is independent of $\epsilon_{j}$ for all $j \neq i$ and of $\theta$. Players then choose simultaneously an action in their action space and payoffs accrue according to $\pi$.

\subsection{The Payoff Functions}

Players are assumed to have a certain degree of exchangeability in their payoffs. They only care about an aggregate, $g$, of opponents' actions. Exchangeability still allows for a wide range of applications that remain convincing in a finite setting. Aggregate $g$ will be specified in more detail later on.

Let $A_{-i}$ be endowed with the product order. Let $d \pi_{i}\left(a_{i}, a_{i}^{\prime}, g\left(a_{-i}\right), \theta\right)=\pi_{i}\left(a_{i}, g\left(a_{-i}\right), \theta\right)-$ $\pi_{i}\left(a_{i}^{\prime}, g\left(a_{-i}\right), \theta\right)$, that is, $d \pi_{i}$ is the difference in player $i$ 's utility to playing $a_{i}$ over $a_{i}^{\prime}$ when facing $a_{-i}$ at state $\theta$. The assumptions on the payoff functions are the following.

\section{Assumption 1 (A1) [Dominance Regions]}

For extreme values of the payoff parameter $\theta$, the extreme actions are strictly dominant: There exist $\underline{\theta}$ and $\bar{\theta}$ in $\mathbb{R}$ with $\underline{\theta}<\bar{\theta}$, where $[\underline{\theta}-\nu, \bar{\theta}+\nu]$ is contained in the interior of the support of $\phi$, such that, for all $i$ and for all $a_{-i}, d \pi_{i}\left(a_{i, m_{i}}, a_{i}, g\left(a_{-i}\right), \theta\right)>0$ if $a_{i} \neq a_{i, m_{i}}$ and $\theta>\bar{\theta}$, and $d \pi_{i}\left(a_{i, 1}, a_{i}, g\left(a_{-i}\right), \theta\right)>0$ if $a_{i} \neq a_{i, 1}$ and $\theta<\underline{\theta}$.

\section{Assumption 2 (A2) [Strategic Complementarities]}

The payoff functions have increasing differences in $\left(a_{i}, a_{-i}\right)$ : For all $a_{i}^{\prime \prime}$ and $a_{i}^{\prime}$ in $A_{i}$ with $a_{i}^{\prime \prime} \succeq_{i} a_{i}^{\prime}, a_{-i}^{\prime \prime}$ and $a_{-i}^{\prime}$ in $A_{-i}$ such that $a_{-i}^{\prime \prime} \succeq a_{-i}^{\prime}, d \pi_{i}\left(a_{i}^{\prime \prime}, a_{i}^{\prime}, g\left(a_{-i}^{\prime \prime}\right), \theta\right) \geq d \pi_{i}\left(a_{i}^{\prime \prime}, a_{i}^{\prime}, g\left(a_{-i}^{\prime}\right), \theta\right)$ for all $\theta \in \mathbb{R}$.

\section{Assumption 3 (A3) [State Monotonicity]}

The payoff functions have strictly increasing differences in $\left(a_{i}, \theta\right)$ : For all $a_{i}^{\prime \prime}$ and $a_{i}^{\prime}$ in $A_{i}$ with $a_{i}^{\prime \prime} \succ_{i} a_{i}^{\prime}, \theta^{\prime \prime}$ and $\theta^{\prime}$ in $[\underline{\theta}, 2 \bar{\theta}-\underline{\theta}]$ such that $\theta^{\prime \prime}>\theta^{\prime}, d \pi_{i}\left(a_{i}^{\prime \prime}, a_{i}^{\prime}, g\left(a_{-i}\right), \theta^{\prime \prime}\right)>$ $d \pi_{i}\left(a_{i}^{\prime \prime}, a_{i}^{\prime}, g\left(a_{-i}\right), \theta^{\prime}\right)$ for all $a_{-i} \in A_{-i}$.

\section{Assumption 4 (A4) [Monotone State Monotonicity]}

The payoff functions exhibit Decreasing (Increasing) State Monotonicity (respectively $(D S M)$ and $(I S M))$ : For all $a_{i}^{\prime \prime}$ and $a_{i}^{\prime}$ in $A_{i}$ with $a_{i}^{\prime \prime} \succ_{i} a_{i}^{\prime}, g^{\prime \prime}$ and $g^{\prime}$ in $\mathcal{H}$ such that $g^{\prime \prime} \succ g^{\prime}, d \pi_{i}\left(a_{i}^{\prime \prime}, a_{i}^{\prime}, g^{\prime \prime}, \theta\right)-d \pi_{i}\left(a_{i}^{\prime \prime}, a_{i}^{\prime}, g^{\prime}, \theta\right)$ is weakly decreasing (or increasing) in $\theta$ on $[\underline{\theta}, 2 \bar{\theta}-\underline{\theta}]$.

\section{Assumption 5 (A5) [Existence of Cutoffs]}


For all $i$, for any $a_{i}^{\prime \prime}, a_{i}^{\prime}$ in $A_{i}$ and for all $a_{-i}$ in $A_{-i}$, there exists $\tilde{\theta} \in[\underline{\theta}, \bar{\theta}]$ such that $d \pi_{i}\left(a_{i}^{\prime \prime}, a_{i}^{\prime}, g\left(a_{-i}\right), \tilde{\theta}\right)=0$.

\section{Assumption 6 (A6) [PAyoff Continuity]}

Each $\pi_{i}\left(a_{i}, g\left(a_{-i}\right), \theta\right)$ is continuous in $\theta$.

We next discuss this set of assumptions. (A1)-(A3) and (A6) are common assumptions in the global game literature. FMP make the same assumptions. CvD only require (A1) and (A6). But CvD's result is limited to $2 \times 2$ games so (A2) is trivially satisfied when there are multiple equilibria, which is the case of interest in selection theory. Further, Morris and Shin [13] develop a model of currency attacks that satisfies (A1), (A2), (A6) and the weak version of (A3).

(A4) and (A5) are however not common in global games. (A4) imposes a monotonicity property on differences. The payoff functions exhibit strictly increasing differences in $\left(a_{i}, \theta\right)$, but this increase in the payoff fades (increases) as $g$ rises. Equivalently, this assumption asserts that the strategic complementarities decrease (increase) with the state, or with the signal when it enters players' payoff directly. Proposition 1 shows it is easy to check whether a twice (or thrice) continuously differentiable function displays (A4).

Proposition 1 Let $\mathcal{A} \subset \mathbb{R}$ and $\mathcal{H} \subset \mathbb{R}$. If a $C^{2}$ function $\pi_{i}: \mathbb{R}^{3} \rightarrow \mathbb{R}$ satisfies $\partial^{2} \pi_{i}\left(a_{i}, g^{\prime \prime}, \theta\right) / \partial a_{i} \partial \theta-\partial^{2} \pi_{i}\left(a_{i}, g^{\prime}, \theta\right) / \partial a_{i} \partial \theta \leq(\geq) 0$ for all $\left(a_{i}, \theta\right) \in \mathbb{R} \times(\underline{\theta}-\epsilon, \bar{\theta}+\epsilon)$ for some $\epsilon>0$ and $g^{\prime \prime}>g^{\prime}$ in $\mathbb{R}$, then its restriction to $\prod_{i} A_{i}$ satisfies (A4). Or, if a $C^{3}$ function $\pi_{i}: \mathbb{R}^{3} \rightarrow \mathbb{R}$ satisfies $\partial^{3} \pi_{i} / \partial a_{i} \partial g \partial \theta \leq(\geq) 0$ for all $\left(a_{i}, g, \theta\right) \in \mathbb{R}^{2} \times(\underline{\theta}-\epsilon, \bar{\theta}+\epsilon)$, then its restriction to $\prod_{i} A_{i}$ satisfies (A4).

Assumption (A5) says that for any pair of actions and any opposing profile, there is a state at which the player is indifferent between them. Note (A5) is redundant in 2-action games since it amounts to "set dominance regions." When there are more than two actions, (A5) rules out, for one, actions that are dominated for all $\theta$. Moreover, requiring in (A5) that cutoff $\tilde{\theta}$ belong to $[\underline{\theta}, \bar{\theta}]$ is not necessary because the existence of such $\tilde{\theta}$ in $\mathbb{R}$ would be sufficient for our purpose. ${ }^{8}$ The following proposition gives some insight into which class of payoff functions satisfy (A5).

Proposition 2 Let $C o\left(A_{i}\right)$ be the convex hull of $A_{i}$. Under (A1) and (A6), if for all $i$, $\pi_{i}\left(a_{i}, g\left(a_{-i}\right), \theta\right)$ is concave in $a_{i} \in C o\left(A_{i}\right)$ for each $a_{-i}, \theta$, then $\pi_{i}$ satisfies (A5) for all $i$.

Proof: Suppose $\pi_{i}$ is concave in $a_{i} \in A_{i}$ for each $a_{-i}$ and $\theta$. Pick $a_{i}^{\prime \prime}$ and $a_{i}^{\prime}$ in $A_{i}$ and suppose $a_{i}^{\prime \prime} \succ_{i} a_{i}^{\prime}$. Choose $a_{-i} \in A_{-i}$ arbitrarily. By (A1), $d \pi_{i}\left(a_{i, m_{i}}, a_{i}^{\prime}, g\left(a_{-i}\right), \theta\right)>0$ for

\footnotetext{
${ }^{8}$ Suppose we only know that all $\tilde{\theta} \equiv \tilde{\theta}\left(a_{i}^{\prime \prime}, a_{i}^{\prime}, a_{-i}\right)$ are in $\mathbb{R}$. Since there are only finitely many, they all are in a compact interval $[l, u]$. Then let $\underline{\theta}_{2}=\min \{\underline{\theta}, l\}$ and $\bar{\theta}_{2}=\max \{\bar{\theta}, u\}$. Therefore, extreme actions are dominant outside $\left[\underline{\theta}_{2}, \bar{\theta}_{2}\right]$ and we can then readjust the new dominance regions to be $\left(-\infty, \underline{\theta}_{2}\right) \cup\left(\bar{\theta}_{2}, \infty\right)$. Now all the cutoffs lie in the non-dominance region as desired. However, by enlarging the non-dominance region, we make (A3) and (A4) stronger.
} 
all $\theta>\bar{\theta}$ and $d \pi_{i}\left(a_{i, 1}, a_{i}^{\prime \prime}, g\left(a_{-i}\right), \theta\right)>0$ for all $\theta<\underline{\theta}$. Since $a_{i, m_{i}} \succeq_{i} a_{i}^{\prime \prime} \succ_{i} a_{i}^{\prime} \succeq_{i} a_{i, 1}$, there exist $\lambda_{1}, \lambda_{2} \in[0,1]$ such that $a_{i}^{\prime \prime}=\lambda_{1} a_{i, m_{i}}+\left(1-\lambda_{1}\right) a_{i}^{\prime}$ and $a_{i}^{\prime}=\lambda_{2} a_{i, 1}+\left(1-\lambda_{2}\right) a_{i}^{\prime \prime}$. Then, by concavity, $d \pi_{i}\left(a_{i}^{\prime \prime}, a_{i}^{\prime}, g\left(a_{-i}\right), \theta\right)>0$ for all $\theta>\bar{\theta}$ and $d \pi_{i}\left(a_{i}^{\prime}, a_{i}^{\prime \prime}, g\left(a_{-i}\right), \theta\right)>0$ for all $\theta<\underline{\theta}$. By $(\mathrm{A} 6)$, the intermediate value theorem implies that there exists $\tilde{\theta} \in[\underline{\theta}, \bar{\theta}]$ such that $\pi_{i}\left(a_{i}^{\prime \prime}, a_{i}^{\prime}, g\left(a_{-i}\right), \tilde{\theta}\right)=0$.

Q.E.D

Finally, I wish to emphasize that the contraction result is, with some qualifications, as general as the previous results of uniqueness for $2 \times 2$ games, because (A4) and (A5) are not needed in this class of games. These assumptions become effective in general finite games, and even though the result becomes more restrictive, it captures interesting applications like currency crises and Diamond's search (See Section 6). In the currency crises model, (A5) will be trivial because there are only two actions, and (A4) will be a sensible property of the exchange rate. In the Diamond's search model, both assumptions will be satisfied in the traditional setting with convex cost functions.

\subsection{The Beliefs}

There are two categories of beliefs each player formulates upon receiving her signal: Those about the state of nature $\theta$, and those about the signal of her opponents $\left(s_{j}\right)_{j \neq i}$. I abuse notation and represent player $i$ 's beliefs about the signal of a subset $J \subset N \backslash\{i\}$ of her opponents by a distribution function $\Omega_{i}\left(s_{J} \mid s_{i}, \nu\right)$. Let $\succeq_{s t}$ stand for the first-order stochastic dominance ordering.

I impose the following assumption on the beliefs.

\section{Assumption 7 (A7) [First-Order stochastic Dominance]}

Let $\Psi_{i}\left(\theta \mid s_{i}, \nu\right)$ be the conditional distribution of $\theta$ given $s_{i}$. For all $i$ and for each $\nu>0$, if $s_{i}^{\prime \prime}>s_{i}^{\prime}$, then $\Psi_{i}\left(\theta \mid s_{i}^{\prime \prime}, \nu\right) \succeq_{s t} \Psi_{i}\left(\theta \mid s_{i}^{\prime}, \nu\right)$.

Unless otherwise specified, assumptions (A1)-(A7) are in effect all throughout this paper.

This condition is the only condition we need on beliefs. While it is not a condition directly on the primitives of the model, it is satisfied by many information structures. For instance, it is satisfied if the prior is uniform and the signaling technologies linear.

(A7) says that a player whose signal increases puts more weight on higher states. Although neither FMP nor CvD make such an assumption on beliefs, it must be satisfied in the limit. Given $\tau_{i}$ and $\epsilon_{i}$ is distributed on a bounded set, then as the error shrinks to zero, player $i$ can restrict the potential values of $\theta$ to be in any neighborhood around $s_{i}$. Therefore, if $i$ 's signal increases from $s_{i}$ to $s_{i}+x^{*}$ with $x^{*}>0$, then there exists $\nu\left(x^{*}\right)>0$ such that $i$ is certain that $\theta$ has also increased for all $\nu<\nu\left(x^{*}\right)$. (A7) is then satisfied whenever $s_{i}^{\prime \prime}>s_{i}^{\prime}+x^{*}$. Therefore, (A7) tends to be satisfied for all $s_{i}^{\prime \prime}$ and $s_{i}^{\prime}$ as $\nu$ goes to zero. 
Now that payoff functions and beliefs have been defined, I spell out the important distinction between private and common values. FMP show that there is continuity between these two versions of the game, and in their methodology, uniqueness in the first one provides sufficient grounds for uniqueness in the second one. ${ }^{9}$

A global game $\Gamma(\nu)$ has either private values or common values. In the private values world, the payoffs directly depend on the type $s_{i}$. This is the case for the investment game in Section 2. The expected payoff for a player $i$ who sees signal $s_{i}$ and is playing $a_{i} \in A_{i}$ against opponents who play according to $\left(a_{j}\left(s_{j}\right)\right)$ is given by:

$$
E \pi_{i}\left(a_{i}, a_{-i}(.), s_{i}\right)=\int_{s_{-i} \in \mathbb{R}^{n-1}} \pi_{i}\left(a_{i}, g\left(a_{-i}\left(s_{-i}\right)\right), s_{i}\right) d \Omega_{i}\left(s_{-i} \mid s_{i}, \nu\right) .
$$

In the common values world, the payoffs depend indirectly on the type $s_{i}$. According to Bayes' rule and given her signal, a player infers the actual state $\theta$ she is in and she constructs beliefs about others' actions. If we denote by $\Omega_{i}\left(s_{-i}, \theta \mid s_{i}, \nu\right)$ the joint conditional distribution of $\left(s_{-i}, \theta\right)$ given $s_{i}$ at $\nu$, the expected payoff for a player $i$ who sees signal $s_{i}$ and is playing $a_{i} \in A_{i}$ against opponents who play according to $\left(a_{j}\left(s_{j}\right)\right)$ is given by:

$$
E \pi_{i}\left(a_{i}, a_{-i}(.), s_{i}\right)=\int_{\theta \in \mathbb{R}} \int_{s_{-i} \in \mathbb{R}^{n-1}} \pi_{i}\left(a_{i}, g\left(a_{-i}\left(s_{-i}\right)\right), \theta\right) d \Omega_{i}\left(s_{-i}, \theta \mid s_{i}, \nu\right) .
$$

\subsection{Increasing Strategies}

In this section, we show that under the above assumptions, it is enough to search for Nash equilibria in increasing strategies because $\Gamma(\nu)$ is a GSC and its extremal equilibria are increasing in the signal. We can then limit our investigation to the class of best-replies that are increasing in the signal.

Definition 1 A normal-form game $\Gamma=\left(N,\left\{\left(S_{i}, u_{i}\right): i \in N\right\}\right)$ is a finite GSC if, for all $i \in N, 1) S_{i}$ is a finite lattice, 2) $s_{i} \mapsto u_{i}\left(s_{i}, s_{-i}\right)$ is supermodular for all $s_{-i} \in S_{-i}$, and $\left(s_{i}, s_{-i}\right) \mapsto u_{i}\left(s_{i}, s_{-i}\right)$ has increasing differences.

For more general definitions of GSC, see for example Milgrom-Roberts [11] or Topkis [21]. The game $\Gamma(\nu)$ is the Bayesian version of a finite GSC, hence it is itself a GSC. By Tarski's theorem, the strategic complementarities yield a greatest and a least equilibria in GSC. Van Zandt and Vives (VZV hereafter) ([24], Theorem 1, p.11) prove that for Bayesian GSC these extremal equilibria are monotone in signal when signals are affiliated. In addition, the best-reply to any increasing strategy (in the signal) must be an increasing strategy. These two results are shown in Proposition 3 which applies both to the common

\footnotetext{
${ }^{9}$ There is only uniqueness in the limit for the common values case. Intuitively, as $\nu$ goes to zero players discard any state $\theta$ that is too far from their signal and so making decision based upon $s_{i}$ being the true state becomes a good approximation. And whatever their prior is, as $\nu$ goes to zero, their signal gets so precise that the private information completely takes over the public information represented by the common prior. Consequently, the common values case converges to the private values case.
} 
and private values. ${ }^{10}$ As a result, if the contraction is established on the set of profiles in increasing strategies, then uniqueness will follow.

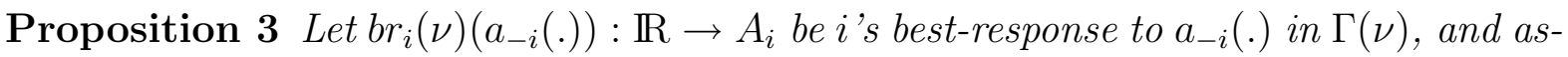
sume (A2), (A3) and (A7). If $a_{j}\left(s_{j}\right)$ is increasing in $s_{j}$ for every $j \neq i$, then $b r_{i}(\nu)\left(a_{-i}().\right)$ is increasing in $s_{i}$. Besides, the greatest (least) equilibrium $\left({\overline{a_{i}}}^{*}\left(s_{i}, \nu\right)\right)\left(\left(\underline{a}_{i}{ }^{*}\left(s_{i}, \nu\right)\right)\right)$ is increasing in $s_{i}$ for all $\nu>0$.

Remark: If the prior is uniform and we assume (A2) and (A3), then the claim of Proposition 3 holds because (A7) is trivially satisfied. Notice also that (A3) implies that the best-replies are almost everywhere functions of the signal, not correspondences.

\section{The Main Result: Contraction of the Best-Reply}

I prove that, under my assumptions, the joint best-reply function in global games is a contraction. I then use this result to show uniqueness.

Definition 2 Let $(X, d)$ be a metric space. If $\xi$ satisfies the condition

$$
d(\xi(x), \xi(y))<d(x, y)
$$

for all $x, y \in X$ with $x \neq y$, then $\xi$ is called a pseudo-contraction (or shrinking map).

Let $\varphi:[\underline{\theta}, \bar{\theta}] \rightarrow[\underline{\theta}, \bar{\theta}]$ be defined by $\varphi\left(c_{1}\right)=\left[c_{1}, \bar{\theta}\right]$ and denote its graph by Gr $\varphi$. Then, let $\varphi^{k}: \operatorname{Gr} \varphi^{k-1} \rightarrow[\underline{\theta}, \bar{\theta}]$ be defined by $\varphi^{k}\left(c_{1}, \ldots, c_{k}\right)=\left[c_{k}, \bar{\theta}\right]$. Let $(\mathcal{C}, d)$ be our metric space where $\mathcal{C}=\prod_{i \in N} \operatorname{Gr} \varphi^{m_{i}-2}$ is the set of profiles in monotone strategies in $\Gamma(\nu)$. For any pair $\left(c^{\prime}, c\right) \in \mathcal{C}^{2}$, let $d\left(c^{\prime}, c\right)=\max _{i \in N} \max _{k_{i}}\left|c_{k_{i}}^{\prime}-c_{k_{i}}\right|$. Denote by $\mathcal{C}_{-i}$ the set of profiles of $i$ 's opponents that are in increasing strategies, and for any $\left(c_{-i}^{\prime}, c_{-i}\right) \in \mathcal{C}_{-i}^{2}$, define $d\left(c_{-i}^{\prime}, c_{-i}\right)=\max _{j \in N \backslash\{i\}} \max _{k_{j}}\left|c_{k_{j}}^{\prime}-c_{k_{j}}\right|$.

\subsection{Private Values Case}

By Proposition 3, we can represent any strategy as a finite sequence of cutoff points. Those cutoff points are called fictitious cutoffs and a formal definition will be given subsequently. We will see that this concept is fundamentally different from the class of real cutoffs. From now on, let player $i$ 's strategy be $\left(c_{i, k_{i}}^{f}\right)_{k_{i}=1}^{m_{i}-1} \in \mathcal{C}_{i}$ where $c_{i, k_{i}}^{f} \in[\underline{\theta}, \bar{\theta}]$ is the threshold below which $i$ plays $a_{i, k_{i}}$ and above which she plays $a_{i, k_{i}+1}$. For notational purposes, we drop the superscript $f$ and we denote these cutoff points by $c_{i, k_{i}}$, or simply $c_{k_{i}}$ when there is no confusion.

\footnotetext{
${ }^{10}$ See Mathevet [10] for a proof
} 
Players only care about an aggregate of opponents' actions. I shall consider two different aggregates: sum and proportion. For all $i \in N$, aggregate sum is defined as follows. For all $a_{-i} \in A_{-i}, \Sigma\left(a_{-i}\right)=\sum_{j \neq i} a_{j}$. Aggregate proportion synthesizes the proportion of opponents playing less than some action $c$. It is defined as follows: For all $a_{-i} \in A_{-i}$, $\Delta\left(a_{-i}\right)=\left(\sum_{j \neq i} 1_{a_{j} \leq c}\right) /(N-1)$ where $c \in \cap_{j \neq i} A_{j} .{ }^{11}$ For sum, the ordering is clear while proportion is endowed with $\succeq_{s t}$, that is, the higher with respect to $>$, the smaller.

As players focus on aggregates that summarize their opponents' play, they only formulate beliefs about what $g$ could be. I abuse notation slightly and refer sometimes to $g$ as an element of $\mathcal{H}$. Let $\Lambda_{i}\left(g \mid c_{-i}, s_{i}, \nu\right)$ be $i$ 's beliefs that the aggregate is strictly less than $g$ when she receives signal $s_{i}$ given her opponents play according to $c_{-i} \in \mathcal{C}_{-i}$. Notice $\Lambda_{i}$ is increasing in $s_{i}$ with respect to $\succeq_{s t}$. To see why, recall that $\epsilon_{j}$ is independent of $s_{i}$ and $\tau_{j}$ is strictly increasing in $\theta$ for all $j \neq i$. Therefore, (A7) implies that player $i$ expects her opponents receive higher signals after hers rises. Since strategies in $\mathcal{C}_{-i}$ are increasing in signals and $g$ is increasing in strategies, we reach the conclusion. ${ }^{12}$

This paper focuses on the study of those particular aggregates, $\Sigma$ and $\Delta$, only because they cover a wide range of applications in global games. The theory developed in this section applies to all aggregates $g$ which, like $\Sigma$ and $\Delta$, are increasing in actions and non-constant.

\subsubsection{Intuition for the Contraction Result}

This section extends the logic of Section 2 and shows the basic intuition behind the whole approach taken in this paper. The point is to extract the general pattern behind Equation 1.

Let $\Gamma(\nu)$ be a global game in private values with $N=\{1,2\}$ and $A_{i}=A=\{0,1\}$ for $i=1,2$. Let $A$ be endowed with the natural order. Let us assume (A1) through (A7). Consider player $j$ has a cutoff strategy, say $c_{j} \in[\underline{\theta}, \bar{\theta}]$. Observe that when player $i$ receives signal $s_{i} \in \mathbb{R}$ and plays $a \in A$, her expected payoff is given by:

$$
E \pi_{i}\left(a, s_{i}\right)=\pi_{i}\left(a, 0, s_{i}\right) \Omega_{i}\left(c_{j} \mid s_{i}, \nu\right)+\pi\left(a, 1, s_{i}\right)\left(1-\Omega_{i}\left(c_{j} \mid s_{i}, \nu\right)\right) .
$$

Since strategies are increasing, player $i$ best-responds to $c_{j}$ when her cutoff is the signal $s_{i}$ such that: $E \pi\left(0, s_{i}\right)=E \pi_{i}\left(1, s_{i}\right)$. This particular signal will be denoted $c_{i}$. If we let

$$
\delta_{i}\left(s_{i}\right)=\pi_{i}\left(1,1, s_{i}\right)-\pi_{i}\left(1,0, s_{i}\right)+\pi_{i}\left(0,0, s_{i}\right)-\pi_{i}\left(0,1, s_{i}\right)
$$

and $r_{i}\left(s_{i}\right)=\pi_{i}\left(1,1, s_{i}\right)-\pi_{i}\left(0,1, s_{i}\right)$, then

$$
\Omega_{i}\left(c_{j} \mid c_{i}, \nu\right) \delta_{i}\left(c_{i}\right)=r_{i}\left(c_{i}\right) .
$$

Dropping the subscript $i$, let $\delta=\delta_{i}\left(c_{i}\right), r=r_{i}\left(c_{i}\right)$ and $V=\Omega_{i}\left(c_{j} \mid c_{i}, \nu\right)$. Graphically, we can represent (4) in the following picture

\footnotetext{
${ }^{11} \cap_{j \neq i} A_{j}$ can be assumed to be nonempty and $c<\max \cup_{i \in N} A_{i}$ as it is the most interesting case.

${ }^{12}$ See Shaked and Shanthikumar [19] (Theorem 4.B.10, p.120) for a formal argument on the closedness of the multivariate stochastic order under diverse operations.
} 


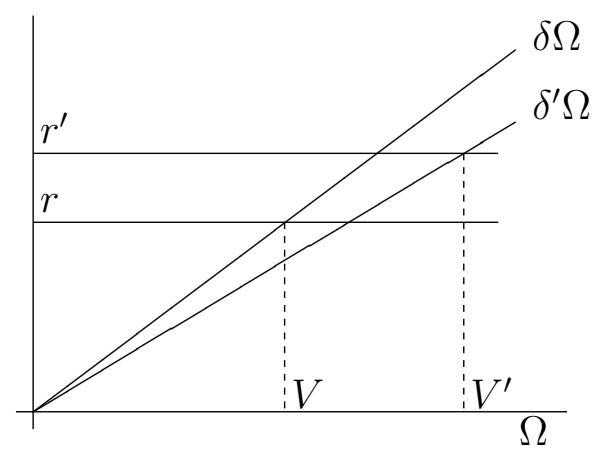

(A2) implies $\delta$ is positive. In addition, both sides of (4) are continuous in $s_{i}$ from (A6) and continuity of $\Lambda_{i}$ in $s_{i}{ }^{13}$ which makes sure the intersection occurs. This unique intersection in the graph determines the value of $\Omega_{i}$ at $c_{i}$.

To establish the contraction property, take any distinct $c_{j}, c_{j}^{\prime} \in[\underline{\theta}, \bar{\theta}]$ and consider the best-replies $c_{i} \equiv b r_{i}\left(c_{j}\right)$ and $c_{i}^{\prime} \equiv b r_{i}\left(c_{j}^{\prime}\right)$. Assume $(D S M)$ and translation increasing beliefs. By way of contradiction, suppose $c_{i}^{\prime} \geq c_{i}+\left|c_{j}^{\prime}-c_{j}\right|$. Let $\delta^{\prime}=\delta_{i}\left(c_{i}^{\prime}\right), r^{\prime}=r_{i}\left(c_{i}^{\prime}\right)$ and $V^{\prime}=\Omega_{i}\left(c_{j}^{\prime} \mid c_{i}^{\prime}, \nu\right)$. Since $c_{i} \equiv b r_{i}\left(c_{j}\right)$, then $\delta V=r$ by $(4)$, but $(D S M)$ and the assumption on beliefs imply $\delta^{\prime} V^{\prime} \leq \delta V$. By (A3), $r^{\prime}>r$. So, put together: $\delta^{\prime} V^{\prime} \leq \delta V=r<r^{\prime}$. This is a contradiction because it violates $\delta^{\prime} V^{\prime}=r^{\prime}$, the optimality condition (4) for $c_{i}^{\prime}$. Therefore, $c_{i}^{\prime}-c_{i}<\left|c_{j}^{\prime}-c_{j}\right|$. Using a similar argument or the monotonicity of $b r_{i}$, we obtain $c_{i}-c_{i}^{\prime}<\left|c_{j}^{\prime}-c_{j}\right|$ and conclude $\left|c_{i}^{\prime}-c_{i}\right|<\left|c_{j}^{\prime}-c_{j}\right|$.

The contradiction is also clear in the graph: Since $r^{\prime}>r$, the intersection must now occur at a (strictly) higher value of $\Omega$ which, by the translation property, is only possible if $c_{i}^{\prime}-c_{i}<\left|c_{j}^{\prime}-c_{j}\right|$.

Translation increasing beliefs are crucial, but note also the role of $(D S M)$. We know $\delta_{i}\left(s_{i}\right)$ is positive by supermodularity which gives players an incentive to respond increasingly when their opponents' actions go up. Nonetheless, this payoff incentive is assumed to die down with the state and so players do not overreact.

Under $(I S M)$, we also reach a contradiction, which shows the overwhelming effect of translation increasing beliefs. But, it is less visible in a graph than before. Indeed, it becomes a matter of which side of (4) rises more. I opted for the more transparent argument here. Further, when beliefs are not translation increasing for every $\nu$ as they were previously, they (uniformly) tend to be as $\nu \rightarrow 0 .{ }^{14}$ This gives rise to several issues that are addressed in Section 7. But the intuition stays the same. The second source of problems is in the extension to many actions. Section 5 deals with this issue and it is treated formally in Section 7.

\footnotetext{
${ }^{13}$ This technical result is proved in Mathevet [10].

${ }^{14}$ See Lemma 2 in Section 7.
} 


\subsubsection{Main Results}

\section{The Contraction}

Here, I generalize the argument just given, but I defer the proofs to Section 7 . First, I define formally the concept of real cutoff.

Definition 3 For player $i$, the real cutoff point between $a_{i, \alpha}, a_{i, \beta} \in A_{i}$ where $\beta>\alpha$ is denoted by $c_{\beta, \alpha}^{r}$ and it is defined as the signal that makes $i$ indifferent between playing $a_{i, \beta}$ and $a_{i, \alpha}: E d \pi_{i}\left(a_{i, \beta}, a_{i, \alpha}, g\left(a_{-i}().\right), c_{\beta, \alpha}^{r}\right)=0$.

The real cutoff between two actions is the only signal at which a player is indifferent between them. Below this signal, the player strictly prefers the smaller one, and above she strictly prefers the bigger one. ${ }^{15}$ But many of these pairwise comparisons are invisible when observing the resulting (increasing) best-response. This gives birth to the fictitious cutoffs that only select those pairwise comparisons that matter to represent the best-reply. I will come back to this issue in some detail in Section 5.

The set of vectors of real cutoffs for player $i$ is $[\underline{\theta}, \bar{\theta}]^{\ell_{i}}$, and it is endowed with metric $\mathrm{t}\left(c_{i}^{\prime r}, c_{i}^{r}\right)=\max _{\left\{\beta, \alpha \in A_{i}: \beta>\alpha\right\}}\left|c_{\beta, \alpha}^{\prime r}-c_{\beta, \alpha}^{r}\right|$ where $c_{i}^{\prime r}, c_{i}^{r}$ are any two elements of the set.

Secondly, I formally define translation increasing beliefs.

Definition 4 For player $i \in N$, beliefs $\Lambda_{i}\left(g \mid c_{-i}, s_{i}, \nu\right)$ are said to be translation increasing at $\nu>0$ if for all $g \in \mathcal{H}, c_{-i} \in \mathcal{C}_{-i}$ and all $x \in[0, \bar{\theta}-\underline{\theta}]^{\left(\sum_{j \neq i} m_{j}-N+1\right)}$ and $x^{*}$ in $[0, \bar{\theta}-\underline{\theta}]$ such that $x^{*} .1 \geq x$, then $\Lambda_{i}\left(g \mid c_{-i}+x, s_{i}+x^{*}, \nu\right) \leq \Lambda_{i}\left(g \mid c_{-i}, s_{i}, \nu\right)$.

Beliefs are translation increasing if, whenever $i$ 's signal increases more than all the opposing cutoffs, then beliefs increase with respect to $\succeq_{s t}$. It plays a very important role in global games as it prevents strategies from getting carried away by the complementarities. When player $i$ increases her strategy more than her opponents, it keeps her from believing that higher values of the aggregate are more likely which moderates her increase. If she put more weight on higher values of $g$, then strategic complementarities would make such an increase optimal.

In addition, this translation property is quite natural because translating the full set of opponents' cutoffs to the right (left) by $x$ should not change the beliefs on average, much less for small $\nu$, if the signal was raised (lowered) by $x$. Then, the definition would follow by first-order stochastic dominance. Notice this characteristic is verified by many distributions with bounded support such as doubly-truncated normals, double exponentials, or some betas centered at $s_{i}{ }^{16}$ and it is a common feature of location-scale families.

The next two results are both contraction results. Recall that $\mathcal{C}_{-i}$ is the set of opposing profiles to player $i$ that are in increasing strategies, and so every profile in this set is

\footnotetext{
${ }^{15}$ There are $\ell_{i} \equiv\left(\begin{array}{l}m_{i} \\ 2\end{array}\right)$ real cutoff points for player $i$.

${ }^{16}$ Normal, double exponential and some Beta distributions are translation increasing and bounded support is not a requirement.
} 
assimilated to a vector of fictitious cutoffs. On the other hand, the superscript $r$ marks the real cutoffs.

Proposition 4 Let $c_{i}^{r}(\nu)$ and $c_{i}^{\prime r}(\nu)$ be respectively the vectors of real cutoffs to $c_{-i}$ and $c_{-i}^{\prime}$ in $\mathcal{C}_{-i}$. If for any $i \in N$, beliefs $\Lambda_{i}\left(\Sigma \mid c_{-i}, s_{i}, \nu\right)$ (or $\Lambda_{i}\left(\Delta \mid c_{-i}, s_{i}, \nu\right)$ ) are translation increasing for each $\nu$, then for all $c_{-i}^{\prime}, c_{-i} \in \mathcal{C}_{-i}$ and all $i \in N, t\left(c_{i}^{\prime r}(\nu), c_{i}^{r}\right.$

$(\nu))<d\left(c_{-i}^{\prime}, c_{-i}\right)$.

\section{Proof: See Section 7.}

Proposition 4 says that every single real cutoff is a contraction of the opposing profile. But this cannot be applied directly to show that the best-response is also a shrinking map. Indeed, we said we could represent any strategy with $m_{i}-1$ cutoff points and yet there are many more real cutoffs. Consequently, a lot of them are vacuous and a selection is performed which leads to Theorem 1. I defer the discussion of this issue to the next section.

Theorem 1 Let $\operatorname{br}(\nu): \mathcal{C} \rightarrow \mathcal{C}$ be the joint best-response function. If for any $i \in N$, beliefs $\Lambda_{i}\left(\Sigma \mid c_{-i}, s_{i}, \nu\right)$ (or $\Lambda_{i}\left(\Delta \mid c_{-i}, s_{i}, \nu\right)$ ) are translation increasing, then $\operatorname{br}(\nu)$ is a pseudo-contraction.

\section{Proof: See Section 7.}

Uniqueness of equilibrium is an important corollary of Theorem 1. We know there exist a greatest and a least equilibrium in $\Gamma(\nu)$ because it is a GSC. However, they cannot be different by the contraction property. FMP's uniqueness result in private values becomes then a consequence of this corollary since uniform prior and linear signaling technologies imply translation increasing beliefs by Corollary 5 of Section 7 .

Definition 5 A Bayesian-Nash equilibrium $\left(a_{i}^{*}().\right)$ is essentially unique if $a_{i}^{*}($.$) is single-$ valued for a.e $s_{i} \in \mathbb{R}$, for all $i \in N$.

Corollary 1 (FMP [4]) If the prior is uniform and the signaling technologies are linear, $\Gamma(\nu)$ has an essentially unique Nash equilibrium for every $\nu>0$.

The seminal work in global games by CvD dealt with $2 \times 2$ games. In this context, (A4) is dispensable and (A5) is trivially satisfied which leads to Proposition 5. The proposition states that the joint best-response in $2 \times 2$ games is contractive, and so there is a unique equilibrium in $2 \times 2$ global games (possibly in the limit) because the joint best-reply is a contraction. This result is established under the traditional assumptions. ${ }^{17}$ Note it is a private values environment, but it is sufficient for uniqueness in the limit (See [4]).

\footnotetext{
${ }^{17}$ Proposition 5 also uses translation increasing beliefs, but as we will see later, beliefs may not satisfy this condition. Nevertheless, there is an analog result of this proposition without the translation hypothesis, along the lines of Theorem 2 and Corollary 2: Under the traditional assumptions, the best-reply in $2 \times 2$ global games is a contraction in the limit.
} 
Proposition 5 Let $\Gamma(\nu)$ be a $2 \times 2$ global game. Under (A1)-(A3) and (A6), if for any $i \in\{1,2\}$, beliefs $\Omega\left(c_{j} \mid s_{i}, \nu\right)$ are translation increasing for each $\nu$, then $\operatorname{br}(\nu)$ is a pseudo-contraction and so an essentially unique equilibrium exists.

The proof is omitted but the argument is simple, ${ }^{18}$ so I outline it here. In Section 4.1.1, note $r_{i}\left(s_{i}\right)=\delta_{i}\left(s_{i}\right)+d \pi_{i}\left(1,0,0, s_{i}\right)$. Hence, $r_{i}$ always increases more than $\delta_{i}$ by (A3). By translation increasing beliefs, the right-hand side of (4) always increases more than the left-hand side whenever $c_{i}^{\prime} \geq c_{i}+\left|c_{j}^{\prime}-c_{j}\right|$. This is a contradiction.

\section{Remarks.}

1. Note that the uniqueness result is also supported by Edelstein's fixed point Theorem $^{19}$ as soon as compactness of $\mathcal{C}$ has been proved. This can be seen by induction since $\mathcal{C}=\prod_{i \in N} \operatorname{Gr} \varphi^{m_{i}-2}$. Clearly, $\varphi$ is closed-valued with compact range and is continuous. As a result $\operatorname{Gr} \varphi$ is closed by the Closed Graph Theorem and trivially, it is bounded. Now, suppose that $\varphi^{k-1}$ has compact graph. Then, $\varphi^{k}$ has compact range as it is continuous. Clearly, $\varphi^{k}$ is closed-valued and so its graph is compact. Therefore, $\operatorname{Gr} \varphi^{m_{i}-2}$ is compact for all $i \in N$. Then $\mathcal{C}$ is compact.

2. Technically, real cutoffs are a well-defined concept. Beliefs $\Omega_{i}\left(\left(a_{j}<s_{j}<b_{j}\right)_{j \neq i} \mid s_{i}\right.$, $\nu$ ) are continuous in the signal for $a<b$ in $\overline{\mathbb{R}}^{n-1} \cdot{ }^{20}$ The finiteness of $A_{i}$ implies that for any $g \in \mathcal{H}$, there are finitely many combinations of opposing profiles that can lead to $g$. Therefore, $\Lambda_{i}\left(g \mid c_{-i}, s_{i}, \nu\right)$ is a finite sum of probabilities of the form $\Omega_{i}\left(\left(a_{j}<s_{j}<b_{j}\right)_{j \neq i} \mid s_{i}, \nu\right)$ so that $\Lambda_{i}$ is continuous as well. Consequently, (A5), (A6), continuity and $\left|A_{-i}\right|<\infty$ ensure that there always exists a real cutoff point in $[\underline{\theta}, \bar{\theta}]$ between two actions whatever the opponents do. The real cutoffs are thus a well-defined concept in $\Gamma(\nu)$.

Although translation increasingness seems like a reasonable requirement, it might be that some information structures do not generate such well-behaved beliefs. In the case of linear signaling technologies, Lemma 1 in Section 7 tells us that this property is satisfied in the limit. In other words, the information structure underlying global games automatically produces translation increasing beliefs as $\nu$ goes to zero. As a result, the best-reply tends to shrink on the whole set of increasing strategies, and every point that falls into the contraction domain at some $\underline{\nu}$ also brings in a neighborhood. This is Theorem 2.

Definition 6 Let $\mathcal{C}_{\nu} \subset \mathcal{C}^{2}$ be the contraction domain of the best-response at $\nu$. That is, for all $\left(c, c^{\prime}\right) \in \mathcal{C}_{\nu}$ with $c \neq c^{\prime}, d\left(b r\left(\nu^{\prime}\right)(c), b r\left(\nu^{\prime}\right)\left(c^{\prime}\right)\right)<d\left(c, c^{\prime}\right)$ for all positive $\nu^{\prime}<\nu{ }^{21}$

\footnotetext{
${ }^{18}$ See Mathevet [10] for the complete proof.

${ }^{19}$ Let $(\mathcal{C}, d)$ be a compact metric space. If $\xi$ is a pseudo-contraction on $\mathcal{C}$ with respect to $d$, then $\xi$ has a unique fixed-point.

${ }^{20}$ See Footnote 13.

${ }^{21}$ Notice that if $\nu^{\prime}<\nu$ then $\mathcal{C}_{\nu} \subset \mathcal{C}_{\nu^{\prime}}$.
} 
Theorem 2 The contraction domain approaches the whole space, that is, $\cup_{\nu>0} \mathcal{C}_{\nu}=\mathcal{C}^{2}$. In addition, for all $\left(c, c^{\prime}\right)$ in $\mathcal{C}^{2}$ with $c \neq c^{\prime}$, there exist a neighborhood $\mathcal{U}$ of $\left(c, c^{\prime}\right)$ and $\underline{\nu}>0$ such that $\mathcal{U} \subset C_{\underline{\nu}}$.

\section{Proof: See Section 7.}

This result has uniqueness in the limit as a corollary. The greatest and the smallest equilibrium must converge towards each other as $\nu$ goes small.

Corollary 2 Let $\bar{e}_{\nu}$ and $\underline{e}_{\nu}$ be respectively the largest and least equilibrium in $\Gamma(\nu)$. Then, $d\left(\bar{e}_{\nu}, \underline{e}_{\nu}\right) \rightarrow 0$ as $\nu \rightarrow 0$ and so there is an essentially unique equilibrium in the limit.

\section{Proof: See Section 7.}

\section{Convergence of Equilibria}

We have learned that under translation increasing beliefs, $\Gamma(\nu)$ only has an essentially unique equilibrium. A natural question is to ask whether this sequence of Nash equilibria converges to some profile as $\nu$ goes to zero. The answer is affirmative given an additional assumption of monotonicity on beliefs. ${ }^{22}$ This is proved in Mathevet [10]. The proof exploits the smoothness of contractive maps and what I call eventually monotone convergence. For what we are concerned with in this paper, FMP [4] proved that the sequence of unique equilibria arising from uniform prior and linear signaling technologies converges to a limit.

\subsection{Common Values Case}

Here players' signals no longer enter their expected payoff directly. I assume signaling technologies are linear. Moreover, the analysis is done under strict first-order stochastic dominance of the beliefs. ${ }^{23}$ This is not more restrictive than (A7) if we assume that for each $\left(s_{i}, \nu\right), \Psi_{i}\left(. \mid s_{i}, \nu\right)$ has full support. Indeed, for $\left(s_{i}, \nu\right), \Psi_{i}:\left[s_{i}-\nu e_{i}, s_{i}+\nu e_{i}\right] \rightarrow[0,1]$ and thus $s_{i}^{\prime \prime} \neq s_{i}^{\prime}$ implies $\Psi_{i}\left(. \mid s_{i}^{\prime \prime}, \nu\right) \neq \Psi_{i}\left(. \mid s_{i}^{\prime}, \nu\right)$. From (A7), we obtain $\succ_{s t}$ by $\succeq_{s t}$ and the non-equality due to the strictly increasing support. For example, $\Psi_{i}$ always has full support when signals are linear and the prior is uniform, since $f_{i}$ has full support by assumption.

Recall that $i$ 's beliefs about $\left(s_{-i}, \theta\right)$ are given by the joint conditional distribution function $\Omega_{i}\left(\left(a_{j}<s_{j}<b_{j}\right)_{j \neq i}, \theta \mid s_{i}, \nu\right)$ for $a<b$ in $\overline{\mathbb{R}}^{n-1}$, which is

$$
\int_{-\infty}^{\theta} \prod_{j \in J}\left(F_{j}\left(\frac{b_{j}-t}{\nu}\right)-F_{j}\left(\frac{a_{j}-t}{\nu}\right)\right) \Psi_{i}^{\prime}\left(t \mid s_{i}, \nu\right) d t .
$$

\footnotetext{
${ }^{22}$ Like (A7), this monotonicity assumption naturally tends to be satisfied in the limit.

${ }^{23}$ This strengthens (A7) to $\succ_{s t}$ where for any distributions $F$ and $G, F \succ_{s t} G$ iff $F \succeq_{s t} G$ and $F \neq G$.
} 
The joint distribution function $\Lambda_{i}\left(g, \theta \mid c_{-i}, s_{i}, \nu\right)^{24}$ represents $i$ 's beliefs about $(g, \theta)$ and it is a finite sum of probabilities of the form $\Omega_{i}\left(\left(a_{j}<s_{j}<b_{j}\right)_{j \neq i}, \theta \mid s_{i}, \nu\right)$. From now on, $\nu$ is supposed to be less than $\eta / e^{*}$ where $\eta>0$. This condition results in no loss of generality since we are interested in uniqueness for $\nu$ small enough. Nevertheless, it ensures that all the real cutoffs are well-defined. I discuss this condition later as a Remark. Finally, we strengthen all the assumptions from (A1) to (A7) by replacing the existing $\underline{\theta}(\bar{\theta})$ with $\underline{\theta}-\eta(\bar{\theta}+\eta)$.

Now, I define the analog of translation increasing beliefs for the common values.

Definition 7 For player $i \in N$, beliefs $\Lambda_{i}\left(g, \theta \mid c_{-i}, s_{i}, \nu\right)$ are said to be increasing in translation (with respect to $\succeq_{s t}$ ) if for all $c_{-i} \in \mathcal{C}_{-i}$, all $x \in[0, \bar{\theta}-\underline{\theta}]^{\left(\sum_{j \neq i} m_{j}-N+1\right)}$ and $x^{*} \in[0, \bar{\theta}-\underline{\theta}]$ such that $x^{*} 1 \geq x$, then $\Lambda_{i}\left(g, \theta \mid c_{-i}+x, s_{i}+x^{*}, \nu\right) \leq \Lambda_{i}\left(g, \theta \mid c_{-i}, s_{i}, \nu\right)$ for all $g \in \mathcal{H}, \theta \in \mathbb{R}$.

Definition 7 says that whenever player $i$ 's signal increases more than does every dimension of the opposing cutoff vector, then her beliefs about the aggregate and the state increase with respect to $\succeq_{s t}$. Interpreted differently, when player $i$ increases her strategy more than her opponent, she thinks that greater aggregative values and higher states are less likely. As actions are strategic complements with one another and with the state, this statistical characteristic plays a moderating role so that strategies are not carried away. Proposition 6 and Lemma 3 in Section 7 show that the information structure of global games implies this form of translation increasing beliefs when the prior is uniform or in the limit (as $\nu$ goes to zero) with a general prior.

The next theorem applies when beliefs are increasing in translation. In this context, there is $\nu$ small enough so that the joint best-response function is a pseudo-contraction. Unlike Theorem 4, we know that a unique equilibrium is actually reached for each $\nu<\eta / e^{*}$; there is no need for a vanishing noise to get uniqueness. This result is noteworthy because the translation assumption is sensible and it is often verified, for instance when the prior is uniform.

Theorem 3 Let $\operatorname{br}(\nu): \mathcal{C} \rightarrow \mathcal{C}$ be the joint best-response function. If for any $i \in N$, beliefs $\Lambda_{i}\left(\Sigma, \theta \mid c_{-i}, s_{i}, \nu\right)$ (or $\Lambda_{i}\left(\Delta, \theta \mid c_{-i}, s_{i}, \nu\right)$ ) are increasing in translation, then for every $\nu<\eta / e^{*}, \operatorname{br}(\nu)$ is a pseudo-contraction and an essentially unique equilibrium exists.

\section{Proof: See Section 7.}

The fundamental result in common values is the next theorem which leads to a unique equilibrium in the limit.

\footnotetext{
${ }^{24}$ This is the probability that the aggregate is strictly less than $g$ and the state less than $\theta$. Continuity of $\Omega_{i}$ in $s_{i}$ can be established in a similar way as before (See Footnote 13). Note the joint distribution function $\Lambda_{i}\left(g, \theta \mid c_{-i}, s_{i}, \nu\right)$ is a finite sum of probabilities of the form $\Omega_{i}\left(\left(a_{j}<s_{j}<b_{j}\right)_{j \neq i}, \theta \mid s_{i}, \nu\right)$ which are continuous in $s_{i}$. Then $\Lambda_{i}$ is also continuous in $s_{i}$. The continuity of $\Lambda_{i}$ in $\left(\theta, s_{i}\right)$ is actually uniform for $s_{i} \in[\underline{\theta}-\eta, \bar{\theta}+\eta]$ for all $i$, as $\theta$ cannot be outside $\left[s_{i}-\nu e^{*}, s_{i}+\nu e^{*}\right]$.
} 
Theorem 4 The contraction domain approaches the whole space, that is, $\cup_{\nu>0} \mathcal{C}_{\nu}=\mathcal{C}^{2}$. In addition, for all $\left(c, c^{\prime}\right)$ in $\mathcal{C}^{2}$ with $c \neq c^{\prime}$, there exist a neighborhood $\mathcal{U}$ of $\left(c, c^{\prime}\right)$ and $\underline{\nu}>0$ such that $\mathcal{U} \subset C_{\underline{\nu}}$.

\section{Proof: See Section 7.}

Corollary 3 (FMP [4]) Let $\bar{e}_{\nu}$ and $\underline{e}_{\nu}$ be respectively the largest and least equilibrium in $\Gamma(\nu)$. Then, $d\left(\bar{e}_{\nu}, \underline{e}_{\nu}\right) \rightarrow 0$ as $\nu \rightarrow 0$ and so there is an essentially unique equilibrium in the limit.

\section{Remarks.}

1. FMP proved that as the signal noise shrinks, the sequence of largest equilibria and the sequence of smallest equilibria in common values converge to the unique outcome of $\Gamma(\nu)$ under private values and uniform prior. ${ }^{25}$ In this last case, the sequence of unique equilibrium converges to a limit, hence all three sequences have the same limit which is the well-known uniqueness prediction of global games.

2. If strict first-order stochastic dominance of $\Psi_{i}$ is weakened to (A7) then best-replies may not be functions. Since global games are GSC, there exists a greatest and a least best-response that are monotone in the signal. It is then possible to show that the contraction domain of those extremal selections approaches the whole space as $\nu \rightarrow 0$ (See Definition 6 and Theorem 4). Therefore, the best-response correspondence is a pseudo-contraction correspondence in the limit. ${ }^{26}$ Uniqueness is reached because those extremal selections become arbitrarily close as $\nu \rightarrow 0$, but they also tend to have a unique fixed point in the limit (See Corollary 3).

3. Notice that results (in private or common values) when $g \equiv \Sigma$ encompass the two-player case with many actions as a situation where each player cares directly about the action taken by her single opponent. In a two-player game with $g \equiv \Delta$, $\mathcal{H}=\{0,1\}$ and each player only cares about a coarse trend of what her opponent plays.

4. The properties that guaranteed existence of real cutoffs in the private values setting are sufficient for small $\nu$ only. To see why, fix $\eta>0$ and let $\nu<\eta / e^{*}$. When player $i$ receives signal $s_{i}$, she knows $\theta \in\left[s_{i}-\nu e_{i}, s_{i}+\nu e_{i}\right]$. Thus, for any $\tilde{\theta} \in \mathbb{R}$, if $s_{i}<\tilde{\theta}-\eta\left(s_{i}>\tilde{\theta}+\eta\right)$, then she is certain that $\theta<(>) \tilde{\theta}$. Therefore, (A5), (A6), $\left|A_{-i}\right|<\infty$, uniform continuity of $\Lambda_{i}$ and $\nu<\eta / e^{*}$ ensure that for all $i \in N$ and $\alpha, \beta \in\left\{1, \ldots, m_{i}\right\}$, there exists $c_{\beta, \alpha}^{r}(\nu) \in[\underline{\theta}-\eta, \bar{\theta}+\eta]$. Since under common values signals do not influence payoffs in a direct manner, they now need to be precise enough for (A5) to act as under private values. Real cutoffs are then a well-defined concept only for $\nu$ small enough.

\footnotetext{
${ }^{25}$ This is Lemma 5 in FMP [4].

${ }^{26}$ See Aliprantis and Border [1] p.552 for a definition of a proper contraction correspondence. Then the definition of a contraction correspondence is the analog version of Definition 2 for correspondences.
} 


\section{On the Fictitious Cutoff Points}

The fictitious cutoffs are the threshold signals that separate an action from its successor, and they are sufficient to represent any increasing (simple) function. Since we only need $m_{i}-1$ cutoffs to represent $i$ 's strategy and we have many more available real cutoffs, we need a way of excluding the dispensable ones. The selection will warrant the separation between Proposition 4 and Theorem 1. Let us start with an example.

ExAmple: Suppose $\Gamma(\nu)$ is a global game with $N=\{1,2\}$ and $A_{1}=A_{2}=\{0,1,2\}$. Further, suppose $\underline{\theta}=0$ and $\bar{\theta}=1$. Here, there will be three real cutoffs: $c_{1,0}^{r}, c_{2,0}^{r}$ and $c_{2,1}^{r}$. But we only need two of them to represent a player's best-response.

For instance, suppose strategy $(0.2,0.8)$ is a best-response to $c_{j} \in \mathcal{C}_{j}$. It consists in playing 0 for signals below $0.2,2$ for signals above 0.8 , and 1 in between. In this case, the first fictitious cutoff, $c_{0}$, that separates 0 and 1 is $0.2=c_{1,0}^{r}$. The second fictitious cutoff, $c_{1}$, that separates 1 and 2 is $0.8=c_{2,1}^{r}$. Now, consider the following best-response $(0.4,0.4)$ to $c_{j}^{\prime} \in \mathcal{C}_{j}$. In this case, the player never plays 1 except possibly on a set of measure zero (when receiving exactly signal 0.4 ). Then the first fictitious cutoff, $c_{0}^{\prime}$, that separates 0 and 1 is $0.4=c_{2,0}^{\prime r}$, but the second fictitious cutoff, $c_{1}^{\prime}$, is also $c_{2,0}^{\prime r}$ since 1 is not played.

Therefore, the fictitious cutoff points take on values of real cutoffs, but they might switch real cutoffs from one opposing profile to the other, which makes it harder to identify any contraction. If each fictitious cutoff was attached to the same real cutoff all the way through, then contraction would follow immediately from Proposition 4.

The next definition is actually the formal derivation of the fictitious cutoff points from a set of real cutoffs. Once I have laid out the definition, I explain the intuition behind Theorem 1 by working through the above example.

Definition 8 We define the fictitious cutoffs inductively. For any $i \in N$, given $c_{-i} \in$ $\mathcal{C}_{-i}$, the greatest fictitious cutoff point, denoted $c_{m_{i}-1}$, is the real cutoff $c_{m_{i}, \alpha}^{r}$ such that: $E d \pi_{i}\left(a_{i, m_{i}}, a_{i}, g\left(c_{-i}\right), s_{i}\right)>0$ for all $a_{i} \neq a_{i, m_{i}}$ and $s_{i}>c_{m_{i}, \alpha}^{r}$, and $E d \pi_{i}\left(a_{i, \alpha}, a_{i}, g\left(c_{-i}\right), s_{i}\right)$ $>0$ for all $a_{i} \neq a_{i, \alpha}$ and $s_{i} \in\left(c_{m_{i}, \alpha}^{r}-\varepsilon, c_{m_{i}, \alpha}^{r}\right)$, for some $\varepsilon>0$. Given $c_{k_{i}}=c_{\beta, \alpha}^{r}$ with $\beta>\alpha$, then the fictitious cutoff that precedes, denoted $c_{k_{i}-1}$, is also equal to $c_{\beta, \alpha}^{r}$ if $k_{i}>\alpha$ or, if $k_{i}=\alpha$, it is the real cutoff $c_{k_{i}, \gamma}^{r}$ with $k_{i}>\gamma$ such that: $E d \pi_{i}\left(a_{k_{i}}, a_{i}, g\left(c_{-i}\right), s_{i}\right)>0$ for all $a_{i} \neq a_{k_{i}}$ and $s_{i} \in\left(c_{k_{i}, \gamma}^{r}, c_{k_{i}, \gamma}^{r}+\varepsilon\right)$, and $E d \pi_{i}\left(a_{i, \gamma}, a_{i}, g\left(c_{-i}\right), s_{i}\right)>0$ for all $a_{i} \neq a_{i, \gamma}$ and $s_{i} \in\left(c_{k_{i}, \gamma}^{r}-\varepsilon, c_{k_{i}, \gamma}^{r}\right)$, for some $\varepsilon>0$.

This defines a map from the real cutoff points to the fictitious cutoffs. For any vector of real cutoffs, there corresponds a unique vector of fictitious cutoffs. Implicitly, we defined the fictitious cutoffs in the context of best-responses only, as they emerge from the real cutoff points.

Now I set out the intuition for the contraction in the context of the example. I only prove that $\left|c_{0}^{\prime}-c_{0}\right|<d\left(c_{j}^{\prime}, c_{j}\right)$ because the proof of $\left|c_{1}^{\prime}-c_{1}\right|<d\left(c_{j}^{\prime}, c_{j}\right)$ is similar.

First, I show $c_{0}^{\prime}-c_{0}<d\left(c_{j}^{\prime}, c_{j}\right)$, that is, $c_{2,0}^{\prime r}-c_{1,0}^{r}<d\left(c_{j}^{\prime}, c_{j}\right)$. For $\beta>\alpha$ recall that once player $i$ prefers $a_{i, \beta}$ to $a_{i, \alpha}$ at signal $s_{i}^{\prime}$, then $a_{i, \beta}$ is strictly preferred to $a_{i, \alpha}$ at any $s_{i}^{\prime \prime}>s_{i}^{\prime}$. 
Therefore, $c_{1,0}^{\prime r} \geq c_{2,0}^{\prime r}$ for if it were not then, when facing $c_{j}^{\prime}$, the player would strictly prefer 1 to 0 for signals in $\left(c_{1,0}^{\prime r}, c_{2,0}^{\prime r}\right)$. But for all signals below $c_{2,0}^{\prime r}$, the player found it optimal to play 0 , so $c_{2,0}^{\prime r}>c_{1,0}^{\prime r}$ cannot be. Then,

$$
c_{0}^{\prime}-c_{0}=c_{2,0}^{\prime r}-c_{1,0}^{r}=c_{2,0}^{\prime r}-c_{1,0}^{\prime r}+c_{1,0}^{\prime r}-c_{1,0}^{r} \leq c_{1,0}^{\prime r}-c_{1,0}^{r}<d\left(c_{j}^{\prime}, c_{j}\right),
$$

where the last inequality follows from Proposition 4 . Secondly, by the same argument as before, $c_{2,0}^{r} \geq c_{1,0}^{r}$. Then

$$
c_{0}-c_{0}^{\prime}=c_{1,0}^{r}-c_{2,0}^{\prime r}=c_{1,0}^{r}-c_{2,0}^{r}+c_{2,0}^{r}-c_{2,0}^{\prime r} \leq c_{2,0}^{r}-c_{2,0}^{\prime r}<d\left(c_{j}^{\prime}, c_{j}\right),
$$

and thus $\left|c_{0}^{\prime}-c_{0}\right|<d\left(c_{j}^{\prime}, c_{j}\right)$.

This argument implies that the map that carries real cutoffs to fictitious cutoffs is continuous in $(\mathcal{C}, d)$. The fictitious cutoffs are proved to contract by showing that they weakly contract as a function of the real cutoffs, which themselves contract as a function of the opponents' strategy.

\section{Examples}

\subsection{Currency Crises}

This is a version of the model by Morris and Shin [13]. Here, a finite number $n$ of speculators decide whether to attack a fixed-exchange regime by selling short one unit of the currency. The current value of the currency is $r^{*}$. The economy is characterized by a state of fundamentals $\theta$ which is distributed according to $\phi$ on a convex subset of $\mathbb{R}$. The currency will float to the shadow rate $\zeta$ if there is no intervention from the monetary authority. The cost of attacking the currency $c(\theta)$ is strictly increasing in $\theta$, reflecting the fact that stronger fundamentals make an attack costlier. Writing 1 for the action "not attack" and 0 for the action "attack," let $\Delta=\left(\sum_{j \neq i} 1_{a_{j}<1}\right) /(n-1)$ for each $i \in N$. The monetary authority defends the currency if this intervention is not too costly. Therefore, the cost of defending the currency is assumed to be increasing in the proportion of speculators who attack, $\Delta$, and decreasing in the state of the fundamentals. There is a minimal proportion of speculators who must attack, $a(\theta)$, for a devaluation to occur. ${ }^{27}$ The payoffs are given by:

$$
\begin{aligned}
& u(1, \Delta, \theta)=0 \\
& u(0, \Delta, \theta)= \begin{cases}-c(\theta), & \text { if } \Delta<a(\theta), \\
r^{*}-\zeta(\theta, \Delta)-c(\theta), & \text { if } \Delta \geq a(\theta),\end{cases}
\end{aligned}
$$

where $a(\theta)$ and $\zeta \equiv \zeta(\theta, \Delta)$ are increasing in $\theta$. Let $\zeta(\theta, \Delta)=r^{*}$ if $\Delta=a(\theta)$, and then suppose that $u$ is continuous in $\theta$ for any $\Delta .^{28}$ Let $M>1$ and $r^{*}-c(\theta)>\zeta(\theta, \Delta)$ for $\Delta>a(\theta)+\frac{1}{M} \cdot{ }^{29}$ Finally, the shadow exchange rate is increasing with $\Delta$ for each $\theta,{ }^{30}$

\footnotetext{
${ }^{27}$ Here, I assume implicitly that both $a(\theta)$ and $n$ make it unnecessary to know whether or not $i$ herself attacks. In other words, given $\Delta$, a player cannot alone spark or prevent the devaluation. For example, assume $a(\mathbb{R}) \subset(-\infty, 0) \cup(1 / n,(n-1) / n) \cup(1, \infty)$.

${ }^{28}$ Note that this condition allows for some discontinuities of $a$ when $\zeta$ and $c$ are continuous.

${ }^{29}$ Suppose $M$ is big enough so the model is interesting for a large range of $\theta$.

${ }^{30}$ This means that $\zeta\left(\theta, \Delta^{\prime}\right)-\zeta\left(\theta, \Delta^{\prime \prime}\right) \geq 0$, whenever $\Delta^{\prime} \succ_{\text {st }} \Delta^{\prime \prime}$ or equivalently $\Delta^{\prime \prime}>\Delta^{\prime}$.
} 
and for any $\Delta^{\prime \prime}>\Delta^{\prime}, \zeta\left(\theta, \Delta^{\prime}\right)-\zeta\left(\theta, \Delta^{\prime \prime}\right)$ is decreasing in $\theta$ which accounts for the larger resistance of the exchange rate to changes in $\Delta$ when fundamentals are stronger. ${ }^{31}$

This is a model where a devaluation does not always benefit the speculators. There exists an interval after $a(\theta)$ where the devaluation is not substantial enough to be profitable. Even if attacking results in a devaluation, it is only optimal after $\Delta$ has passed some threshold $a(\theta)+\frac{1}{M}$.

This game satisfies the standard assumptions of global games, (A1) through (A3) and (A6). Also notice the traditional tripartite division of fundamentals. If $\theta<a^{-1}\left(-\frac{1}{M}\right)$, each player has a dominant strategy to attack and if $\theta>a^{-1}(1)$, each of them has a dominant strategy not to attack. In addition, there is an interval in $\left(a^{-1}\left(-\frac{1}{M}\right), a^{-1}(1)\right)$ where the two symmetric profiles are both equilibria. There are only two actions so that (A5) is automatically satisfied, and $(D S M)$ also holds.

\subsection{Diamond's Search Model}

This is a version of the Diamond-type search model of Milgrom and Roberts [11]. There are a finite number of players $\{1, \ldots, n\}$ who exert effort $a_{i} \in A_{i} \equiv\{0, \ldots, \bar{a}\}$ searching for trading partners. The probability of any trader to find a partner is proportional to her own effort and the sum of the efforts of the others denoted $\Sigma_{-i}$. The economy is characterized by a state of the fundamentals $\theta$ which is distributed according to $\phi$ on a convex subset of $\mathbb{R}$, and each player receives a private signal about $\theta$ with the properties described earlier. The positive cost to individual $i$ for exerting a level of search $a_{i}$ is $c_{i}\left(a_{i}, \theta\right)$ and so her payoff is defined by:

$$
\pi_{i}\left(a_{i}, \Sigma_{-i}, \theta\right)=\alpha a_{i} \Sigma_{-i}-c_{i}\left(a_{i}, \theta\right)
$$

where $\alpha>0$. Since $\partial^{2} \pi_{i} / \partial a_{i} \partial \Sigma=\alpha$ for $i \neq j$, this is a supermodular game in $\left(a_{i}, \Sigma\right)$ and so (A2) is satisfied. For simplicity, cost function $c_{i}$ is assumed to be continuous in $\theta$ which verifies (A6), and it is $C^{2}$ on $C o\left(A_{i}\right)$ for each $\theta$. Moreover, $c_{i}$ has strictly decreasing differences in $\left(a_{i}, \theta\right)$, and in particular, there exist $\underline{\theta}$ and $\bar{\theta}$ with $\underline{\theta}<\bar{\theta}$ such that for all $a_{i} \in A_{i}, c_{i}^{\prime}\left(a_{i}, \theta\right)>\alpha(n-1)(\bar{a}+\varepsilon)$ for some $\varepsilon>0$ and all $\theta<\underline{\theta}$, and $c_{i}^{\prime}\left(a_{i}, \theta\right)<0$ for all $\theta>\bar{\theta}$. For example, costs may be decreasing when learning is taken into account. The state of the world $\theta$ could summarize knowledge in the economy and as $\theta$ increases the marginal cost strictly decreases which accounts for (A3). In particular, there exists a state $\bar{\theta}$ from which learning (by doing) is so high that each unit produced decreases the cost. As a result, (A1) holds and since $c_{i}$ is traditionally convex in $a_{i}$, then by Proposition 2, (A5) is satisfied. Finally, Proposition 1 establishes (A4).

The theory of Section 4 then applies. Consider the symmetric version of the game. Notice that there exists $\tilde{\theta}>\underline{\theta}$ such that $c(., \theta)$ is strictly increasing in $a_{i}$ for all $\theta \in(\underline{\theta}, \tilde{\theta})$ because $A_{i}$ is finite, $c^{\prime}\left(a_{i}, \theta\right)>\alpha(n-1)(\bar{a}+\varepsilon)$ and $c^{\prime}$ is continuous. Therefore, $a_{i}=0$

\footnotetext{
${ }^{31}$ Morris and Shin [13] originally assumed $\zeta \equiv \zeta(\theta)$ is increasing in $\theta$ which satisfies all my requirements for $\zeta$ except $\zeta(\theta, \Delta)=r^{*}$ if $\Delta=a(\theta)$; but their setting does not allow continuity of $u$ in $\theta$ for all $\Delta$.
} 
for all $i$ is an equilibrium. Since $c$ is smooth and convex, if $A_{i}$ is rich enough, then there is also a symmetric equilibrium where effort levels across players are the same. This equilibrium is derived from the optimality condition in the case where $A_{i}=[0, \bar{a}]$ : $\alpha(n-1) a^{*}=c^{\prime}\left(a^{*}, \theta\right)$. Consequently, there is an interval in $(\underline{\theta}, \tilde{\theta})$ for which there are multiple equilibria.

\section{Proofs}

Without further notice, the proofs are given assuming $(D S M)$. Most of the proofs under $(I S M)$ can be found in Mathevet [10]. ${ }^{32}$

Proof of Proposition 4: I prove the result for $g \equiv \Sigma$ as the same argument clearly applies to $\Delta$.

Let $\bar{\Sigma}_{-i}=\sum_{j \neq i} a_{j, m_{j}}$ and $\rho: \mathcal{H} \rightarrow \mathcal{H}$ be the successor function defined for all $\Sigma \in \mathcal{H}$ by $\rho(\Sigma)=\min \left\{\Sigma^{\prime} \in \mathcal{H}: \Sigma^{\prime} \succ \Sigma\right\}$. Let $\underline{\Sigma}_{-i}=\sum_{j \neq i} a_{j, 1}$. Since there is no confusion, the subscript is dropped for the extremal values of the aggregate. Take any $c_{-i}^{\prime}, c_{-i} \in \mathcal{C}_{-i}$ with $c_{-i}^{\prime} \neq c_{-i}$. From Equation 2, we can write $i$ 's expected utility to playing $a_{i}$ when the other players play according to $c_{-i}$ :

$$
\begin{aligned}
E \pi_{i}\left(a_{i}, c_{-i}, s_{i}\right)= & \Lambda_{i}\left(\rho(\underline{\Sigma}) \mid c_{-i}, s_{i}, \nu\right) \pi_{i}\left(a_{i}, \underline{\Sigma}, s_{i}\right)+\sum_{\{\Sigma: \bar{\Sigma}>\Sigma>\underline{\Sigma}\}}\left(\Lambda_{i}\left(\rho(\Sigma) \mid c_{-i}, s_{i}, \nu\right)\right. \\
& \left.-\Lambda_{i}\left(\Sigma \mid c_{-i}, s_{i}, \nu\right)\right) \pi_{i}\left(a_{i}, \Sigma, s_{i}\right)+\left(1-\Lambda_{i}\left(\bar{\Sigma} \mid c_{-i}, s_{i}, \nu\right)\right) \pi_{i}\left(a_{i}, \bar{\Sigma}, s_{i}\right)
\end{aligned}
$$

which can be rewritten,

$$
\begin{aligned}
E \pi_{i}\left(a_{i}, c_{-i}, s_{i}\right)= & \pi_{i}\left(a_{i}, \bar{\Sigma}, s_{i}\right)+\sum_{\{\Sigma: \bar{\Sigma}>\Sigma \geq \Sigma} \Lambda_{i}\left(\rho(\Sigma) \mid c_{-i}, s_{i}, \nu\right)\left(\pi_{i}\left(a_{i}, \Sigma, s_{i}\right)-\right. \\
& \left.-\pi_{i}\left(a_{i}, \rho(\Sigma), s_{i}\right)\right) .
\end{aligned}
$$

Now, pick $\beta, \alpha \in\left\{1, \ldots, m_{i}\right\}$ such that $\beta>\alpha$. For any $\Sigma, \Sigma^{\prime}$ in $\mathcal{H}$ and signal $s_{i}$, let

$$
\delta\left(\Sigma, \Sigma^{\prime}, s_{i}\right)=d \pi_{i}\left(a_{i, \alpha}, a_{i, \beta}, \Sigma, s_{i}\right)-d \pi_{i}\left(a_{i, \alpha}, a_{i, \beta}, \Sigma^{\prime}, s_{i}\right)
$$

By definition, $c_{\beta, \alpha}^{r}$ is the signal $s_{i}$ that verifies $E d \pi_{i}\left(a_{i, \alpha}, a_{i, \beta}, c_{-i}, s_{i}\right)=0$ which by $(7)$ is equivalent to the signal $s_{i}$ such that

$$
\sum_{\{\Sigma: \bar{\Sigma}>\Sigma \geq \Sigma\}} \Lambda_{i}\left(\rho(\Sigma) \mid c_{-i}, s_{i}, \nu\right) \delta\left(\Sigma, \rho(\Sigma), s_{i}\right)=d \pi_{i}\left(a_{i, \beta}, a_{i, \alpha}, \bar{\Sigma}, s_{i}\right)
$$

Denote by $l\left(c_{-i}, s_{i}\right)$ and $r\left(s_{i}\right)$ respectively the left-hand side and right-hand side in (8). Let $x_{j, k_{j}}=\left|c_{j, k_{j}}^{\prime}-c_{j, k_{j}}\right|, x=\left(x_{j, k_{j}}\right)_{j \neq i}$ and $x^{*} \geq \max _{j \neq i} \max _{k_{j}} x_{j, k_{j}}$. Suppose by way of contradiction that $c_{\beta, \alpha}^{\prime r}=c_{\beta, \alpha}^{r}+x^{*}$. By definition, $\left(c_{-i}^{\prime}, c_{\beta, \alpha}^{\prime r}\right)$ must also satisfy (8). By translation increasing beliefs, $\Lambda_{i}\left(\rho(\Sigma) \mid c_{-i}+x, c_{\beta, \alpha}^{r}+x^{*}, \nu\right) \leq \Lambda_{i}\left(\rho(\Sigma) \mid c_{-i}, c_{\beta, \alpha}^{r}, \nu\right)$ for all

\footnotetext{
${ }^{32}$ The other proofs under $(I S M)$ are available to the reader upon request.
} 
$\Sigma \in \mathcal{H}$, and thus $\Lambda_{i}\left(\rho(\Sigma) \mid c_{-i}^{\prime}, c_{\beta, \alpha}^{\prime r}, \nu\right) \leq \Lambda_{i}\left(\rho(\Sigma) \mid c_{-i}, c_{\beta, \alpha}^{r}, \nu\right)$ for all $\Sigma .{ }^{33}$ Supermodularity of $\pi_{i}$ in $\left(a_{i}, \Sigma\right)$ implies that $\delta($.$) is always positive, but \delta\left(\Sigma, \rho(\Sigma), c_{\beta, \alpha}^{\prime r}\right) \leq \delta\left(\Sigma, \rho(\Sigma), c_{\beta, \alpha}^{r}\right)$ for all $\Sigma$ by (A4). By strictly increasing differences, $r\left(s_{i}\right)$ is strictly increasing in $s_{i}$. Therefore, $l\left(c_{-i}^{\prime}, c_{\beta, \alpha}^{\prime r}\right) \leq l\left(c_{-i}, c_{\beta, \alpha}^{r}\right)=r\left(c_{\beta, \alpha}^{r}\right)<r\left(c_{\beta, \alpha}^{\prime r}\right)$. Since $\left(c_{-i}^{\prime}, c_{\beta, \alpha}^{\prime r}\right)$ does not satisfy (8), it is a contradiction. Consequently, $c_{\beta, \alpha}^{\prime r}-c_{\beta, \alpha}^{r}<x^{*}$. By a similar argument but starting from $\left(c_{-i}^{\prime}, c_{\beta, \alpha}^{\prime r}\right)$, we obtain $\left|c_{\beta, \alpha}^{\prime r}-c_{\beta, \alpha}^{r}\right|<x^{*}$. Since $\beta, \alpha$ were chosen arbitrarily in a finite set and $x^{*}$ was any real number greater or equal to $d\left(c_{-i}^{\prime}, c_{-i}\right)$, we get $\mathrm{t}\left(c_{i}^{\prime r}(\nu), c_{i}^{r}(\nu)\right)<d\left(c_{-i}^{\prime}, c_{-i}\right)$.

Q.E.D

Proof of Theorem 1: I want to show first that $b r_{i}(\nu): \mathcal{C}_{-i} \rightarrow \mathcal{C}_{i}$ is a shrinking map for all $i \in N$ and for each $\nu$. Pick an arbitrary $\nu>0$ that is implicit all throughout, and drop the subscript $i$ when it is obvious. By Proposition 4, we know that for all distinct $c_{-i}^{\prime}, c_{-i} \in \mathcal{C}_{-i}$ and all $i \in N, \mathrm{t}\left(c_{i}^{\prime r}, c_{i}^{r}\right)<d\left(c_{-i}^{\prime}, c_{-i}\right)$. Let $b r_{i}\left(c_{-i}\right)=\left(c_{k_{i}}\right)_{k_{i}}$ and $b r_{i}\left(c_{-i}^{\prime}\right)=\left(c_{k_{i}}^{\prime}\right)_{k_{i}}$. It is to be shown that $\left|c_{\beta, \alpha}^{\prime r}-c_{\beta, \alpha}^{r}\right|<d\left(c_{-i}^{\prime}, c_{-i}\right)$ for all $\beta, \alpha, i$ implies $\left|c_{k_{i}}^{\prime}-c_{k_{i}}\right|<d\left(c_{-i}^{\prime}, c_{-i}\right)$ for all $k_{i}$ and $i \in N$.

I prove this result by induction for an arbitrary pair $\left(c_{-i}^{\prime}, c_{-i}\right) \in \mathcal{C}_{-i}^{2}$ of distinct elements. First, I show it is true for the greatest fictitious cutoff point. Recall that (A1) implies that $a_{m_{i}}$ must be played in any best-response. Suppose that $c_{m_{i}-1}=c_{m_{i}, s}^{r}$ and $c_{m_{i}-1}^{\prime}=c_{m_{i}, t}^{\prime r}$ for some $s, t \in\left\{1, \ldots, m_{i}-1\right\}$. Then

$$
c_{m_{i}-1}^{\prime}-c_{m_{i}-1}=c_{m_{i}, t}^{\prime r}-c_{m_{i}, s}^{r}=c_{m_{i}, t}^{\prime r}-c_{m_{i}, t}^{r}+c_{m_{i}, t}^{r}-c_{m_{i}, s}^{r}
$$

By Proposition 4, $c_{m_{i}, t}^{r}-c_{m_{i}, t}^{r}<d\left(c_{-i}^{\prime}, c_{-i}\right)$. By Definition 8, $c_{m_{i}, t}^{r}-c_{m_{i}, s}^{r} \leq 0$ because $c_{m_{i}-1}=c_{m_{i}, s}^{r}$ means that $a_{m_{i}}$ is played right after $a_{s}$ in the best-reply, and thus the real cutoff between $a_{m_{i}}$ and $a_{t}$ must lie before $c_{m_{i}, s}^{r}$. As a result, $c_{m_{i}-1}^{\prime}-c_{m_{i}-1}<d\left(c_{-i}^{\prime}, c_{-i}\right)$. Clearly, we can apply the same argument to show $c_{m_{i}-1}-c_{m_{i}-1}^{\prime}<d\left(c_{-i}^{\prime}, c_{-i}\right)$ and so $\left|c_{m_{i}-1}^{\prime}-c_{m_{i}-1}\right|<d\left(c_{-i}^{\prime}, c_{-i}\right)$.

Now suppose that $\left|c_{k_{i}}^{\prime}-c_{k_{i}}\right|<d\left(c_{-i}^{\prime}, c_{-i}\right)$ for $k_{i}$, and I prove that $\left|c_{k_{i}-1}^{\prime}-c_{k_{i}-1}\right|<$ $d\left(c_{-i}^{\prime}, c_{-i}\right)$.

CASE 1: Action $a_{k_{i}}$ is played both under $b r_{i}\left(c_{-i}\right)$ and $b r_{i}\left(c_{-i}^{\prime}\right)$. Notice this is similar to the case of the greatest fictitious cutoff and so the proof is analogous.

CASE 2: Action $a_{k_{i}}$ is played neither under $b r_{i}\left(c_{-i}\right)$ nor $b r_{i}\left(c_{-i}^{\prime}\right)$. Then, $c_{k_{i}-1}=c_{k_{i}}$ and $c_{k_{i}-1}^{\prime}=c_{k_{i}}^{\prime}$, and so $\left|c_{k_{i}-1}^{\prime}-c_{k_{i}-1}\right|<d\left(c_{-i}^{\prime}, c_{-i}\right)$ by induction hypothesis.

CASE 3A: Action $a_{k_{i}}$ is not played under $b r_{i}\left(c_{-i}\right)$ but it is under $b r_{i}\left(c_{-i}^{\prime}\right)$. Then, $c_{k_{i}-1}=c_{k_{i}}$ and so $c_{k_{i}-1}^{\prime}-c_{k_{i}-1}=c_{k_{i}-1}^{\prime}-c_{k_{i}}=c_{k_{i}-1}^{\prime}-c_{k_{i}}^{\prime}+c_{k_{i}}^{\prime}-c_{k_{i}}$. Notice $c_{k_{i}}^{\prime}-c_{k_{i}}<d\left(c_{-i}^{\prime}, c_{-i}\right)$ by induction hypothesis and $c_{k_{i}-1}^{\prime}-c_{k_{i}}^{\prime} \leq 0$ since the best-response is increasing in the signal. Therefore, $c_{k_{i}-1}^{\prime}-c_{k_{i}-1}<d\left(c_{-i}^{\prime}, c_{-i}\right)$.

CASE 3B: By a similar argument, if action $a_{k_{i}}$ is not played under $b r_{i}\left(c_{-i}^{\prime}\right)$ but it is under $b r_{i}\left(c_{-i}\right), c_{k_{i}-1}-c_{k_{i}-1}^{\prime}<d\left(c_{-i}^{\prime}, c_{-i}\right)$.

CASE 4A: Action $a_{k_{i}}$ is played under $b r_{i}\left(c_{-i}^{\prime}\right)$ but it is not played under $b r_{i}\left(c_{-i}\right)$. Then,

\footnotetext{
${ }^{33}$ Notice $\Lambda_{i}\left(\rho(\Sigma) \mid c_{-i}^{\prime}, c_{\beta, \alpha}^{\prime r}, \nu\right) \leq \Lambda_{i}\left(\rho(\Sigma) \mid c_{-i}+x, c_{\beta, \alpha}^{\prime r}, \nu\right)$ because $\Sigma$ is increasing in the players' actions and, when player $i$ 's opponents lower all their cutoffs player $i$ believes higher actions are more likely and so are greater sums.
} 
$c_{k_{i}-1}^{\prime}=c_{k_{i}, t}^{\prime r}$ and $c_{k_{i}-1}=c_{k_{i}}=c_{s, w}^{r}$ for some $s, t, w \in\left\{1, \ldots, m_{i}\right\}$ where $s>k_{i}$ and $w<k_{i}$ (since $a_{k_{i}}$ is not played). Note that $c_{s, w}^{r}-c_{k_{i}, w}^{r} \leq 0$ as it is optimal to play $a_{w}$ until $c_{s, w}^{r}$ and so $a_{k_{i}} \succ_{i} a_{w}$ must occur for higher signals than $c_{s, w}^{r}$. Suppose first that $w=t$. Since $c_{k_{i}-1}-c_{k_{i}-1}^{\prime}=c_{s, w}^{r}-c_{k_{i}, w}^{r}+c_{k_{i}, w}^{r}-c_{k_{i}, w}^{r}$, then Proposition 4 leads to $c_{k_{i}-1}-c_{k_{i}-1}^{\prime}<d\left(c_{-i}^{\prime}, c_{-i}\right)$. Now suppose $w \neq t$. Then $c_{k_{i}-1}-c_{k_{i}-1}^{\prime}=c_{s, w}^{r}-c_{k_{i}, t}^{\prime r}$. Recall $c_{k_{i}, w}^{r} \geq c_{s, w}^{r}$, and notice $c_{k_{i}, w}^{\prime r} \leq c_{k_{i}, t}^{\prime r}$ because it is a best-response to play $a_{k_{i}}$ (in some interval starting) from $c_{k_{i}, t}^{\prime r}$ and so $a_{k_{i}} \succ_{i} a_{w}$ must occur before that signal $c_{k_{i}, t}^{\prime r}$. As a result, $c_{k_{i}-1}-c_{k_{i}-1}^{\prime}=c_{s, w}^{r}-c_{k_{i}, t}^{\prime r} \leq c_{k_{i}, w}^{r}-c_{k_{i}, w}^{\prime r}$. By Proposition $4, c_{k_{i}, w}^{r}-c_{k_{i}, w}^{\prime r}<d\left(c_{-i}^{\prime}, c_{-i}\right)$ and so $c_{k_{i}-1}-c_{k_{i}-1}^{\prime}<d\left(c_{-i}^{\prime}, c_{-i}\right)$.

CASE 4B: By a similar argument, if action $a_{k_{i}}$ is played under $b r_{i}\left(c_{-i}\right)$ but it is not under $b r_{i}\left(c_{-i}^{\prime}\right)$, then $c_{k_{i}-1}^{\prime}-c_{k_{i}-1}<d\left(c_{-i}^{\prime}, c_{-i}\right)$.

Put Cases 3A and 4A (3B and 4B) together to obtain: $\left|c_{k_{i}-1}^{\prime}-c_{k_{i}-1}\right|<d\left(c_{-i}^{\prime}, c_{-i}\right)$, which completes the induction part of the proof. Therefore, $\left|c_{k_{i}}^{\prime}-c_{k_{i}}\right|<d\left(c_{-i}^{\prime}, c_{-i}\right)$ for all $k_{i}$, and so best-response $b r_{i}: \mathcal{C}_{-i} \rightarrow \mathcal{C}_{i}$ shrinks for all $i \in N$. For all distinct $c^{\prime}, c \in \mathcal{C}$ and for all $i \in N$ such that $c_{-i}^{\prime} \neq c_{-i}, \max _{k_{i}}\left|c_{k_{i}}^{\prime}-c_{k_{i}}\right|<d\left(c_{-i}^{\prime}, c_{-i}\right) \leq d\left(c^{\prime}, c\right)$ which implies that $\max _{i \in N} \max _{k_{i}}\left|c_{k_{i}}^{\prime}-c_{k_{i}}\right|<d\left(c^{\prime}, c\right)$. Equivalently, $d\left(b r\left(c^{\prime}\right), b r(c)\right)<d\left(c^{\prime}, c\right)$ for all $c^{\prime}, c \in \mathcal{C}$ with $c^{\prime} \neq c$.

Q.E.D

The next Lemma draws its main idea and technicalities from Lemma 4 in FMP [4]. Lemma 1 says that beliefs $\Omega_{i}$ tend to be translation invariant in the limit and this is achieved uniformly in $\nu$ for all parameters. Denote

$$
\rho_{x^{*}}(\nu)=\max _{s_{i} \in[\underline{\theta}, \bar{\theta}]} \frac{\max _{e \in\left[-e_{i}, e_{i}\right]} \phi\left(s_{i}+x^{*}-\nu e\right)}{\min _{e \in\left[-e_{i}, e_{i}\right]} \phi\left(s_{i}+x^{*}-\nu e\right)},
$$

where $x^{*} \in[0, \bar{\theta}-\underline{\theta}]$. Define $\varepsilon_{x^{*}}(\nu)=\max \left\{1-1 / \rho_{x^{*}}(\nu), \rho_{x^{*}}(\nu)-1\right\}$.

Lemma 1 Let $J \subset N \backslash\{i\}$, and let $K=\left[\underline{\theta}-\nu e^{*}, 2 \bar{\theta}-\underline{\theta}+\nu e^{*}\right]$ be contained in the interior of the support of $\phi$. Let $i \in N$ and $x^{*} \in[\overline{0}, \bar{\theta}-\underline{\theta}]$. Denote

$$
\kappa_{x^{*}}\left(c_{-i}, s_{i}, \nu\right)=\int_{-\infty}^{\infty} \frac{1}{\nu} \prod_{j \in J} F_{j}\left(\frac{c_{j}+x^{*}-\theta}{\nu}\right) f_{i}\left(\frac{s_{i}+x^{*}-\theta}{\nu}\right) d \theta .
$$

Then, $\varepsilon_{x^{*}}(\nu) \rightarrow 0$ and,

$$
\left|\Omega_{i}\left(c_{-i}+x^{*} \mathbf{1} \mid s_{i}+x^{*}, \nu\right)-\kappa_{x^{*}}\left(c_{-i}, s_{i}, \nu\right)\right|<\varepsilon_{x^{*}}(\nu), \text { for all } s_{i} \in[\underline{\theta}, \bar{\theta}], c_{-i} \in \mathcal{C}_{-i} .
$$

Proof: Since the support of $\phi$ includes $K, \phi$ has a strictly positive minimum over this interval. Since $\phi$ is continuous, then by Dini's theorem ${ }^{34} \max _{e \in\left[-e_{i}, e_{i}\right]} \phi\left(s_{i}+x^{*}-\nu e\right)-$ $\min _{e \in\left[-e_{i}, e_{i}\right]} \phi\left(s_{i}+x^{*}-\nu e\right)$ converges to 0 as $\nu \rightarrow 0$ uniformly for $s_{i} \in[\underline{\theta}, \bar{\theta}]$. Therefore,

$$
\rho_{x^{*}}(\nu)=\max _{s_{i} \in[\underline{\theta}, \bar{\theta}]}\left(1+\frac{\max _{e \in\left[-e_{i}, e_{i}\right]} \phi\left(s_{i}+x^{*}-\nu e\right)-\min _{e \in\left[-e_{i}, e_{i}\right]} \phi\left(s_{i}+x^{*}-\nu e\right)}{\min _{e \in\left[-e_{i}, e_{i}\right]} \phi\left(s_{i}+x^{*}-\nu e\right)}\right) \rightarrow 1
$$

\footnotetext{
${ }^{34}$ If a net of continuous real functions on a compact space converges monotonically to a continuous function pointwise, then the net converges uniformly.
} 
as $\nu \rightarrow 0$. Now, notice that for all parameters in their respective sets,

$$
\frac{1}{\rho_{x^{*}}(\nu)} \leq \frac{\phi\left(s_{i}+x^{*}-\nu z\right)}{\int_{-\infty}^{\infty} f_{i}(z) \phi\left(s_{i}+x^{*}-\nu z\right) d z} \leq \rho_{x^{*}}(\nu),
$$

and thus $\phi() /.\left(\int f_{i}(.) \phi().\right)$ uniformly converges to 1 as $\nu \rightarrow 0$. The $\operatorname{cdf} \Omega_{i}$ is given by

$$
\Omega_{i}\left(c_{-i}+x^{*} \mathbf{1} \mid s_{i}+x^{*}, \nu\right)=\int_{-\infty}^{\infty} \prod_{j \in J} F_{j}\left(\frac{c_{j}+x^{*}-\theta}{\nu}\right) \frac{f_{i}\left(\frac{s_{i}+x^{*}-\theta}{\nu}\right) \phi(\theta)}{\int_{-\infty}^{\infty} f_{i}\left(\frac{s_{i}+x^{*}-\theta}{\nu}\right) \phi(\theta) d \theta} d \theta
$$

I make the following change of variables: $z=\frac{s_{i}+x^{*}-\theta}{\nu}$ and rewrite (10) as

$$
\int_{-e_{i}}^{e_{i}} \prod_{j \in J} F_{j}\left(\frac{c_{j}-s_{i}+\nu z}{\nu}\right) \frac{f_{i}(z) \phi\left(s_{i}+x^{*}-\nu z\right)}{\int_{-e_{i}}^{e_{i}} f_{i}(z) \phi\left(s_{i}+x^{*}-\nu z\right) d z} d z
$$

Consequently,

$$
\begin{gathered}
\left|\Omega_{i}\left(c_{-i}+x^{*} \mathbf{1} \mid s_{i}+x^{*}, \nu\right)-\int_{-e_{i}}^{e_{i}} \prod_{j \in J} F_{j}\left(\frac{c_{j}-s_{i}+\nu z}{\nu}\right) f_{i}(z) d z\right| \\
\leq \int_{-e_{i}}^{e_{i}} f_{i}(z)\left|\varepsilon_{x^{*}}(\nu)\right| d z \leq \varepsilon_{x^{*}}(\nu) \rightarrow 0 \text { as } \nu \rightarrow 0 \text { by }(9) .
\end{gathered}
$$

The claim is proved.

Q.E.D

The next Lemma shows that beliefs about the aggregate tend to be translation increasing in the limit and this is achieved uniformly in $\nu$ for all parameters. In the proof, notice that I only make use of the monotonicity of $\Sigma$ and the proof would go similarly with any increasing $g$.

Lemma 2 Let $K=\left[\underline{\theta}-\nu e^{*}, 2 \bar{\theta}-\underline{\theta}+\nu e^{*}\right]$ be contained in the interior of the support of $\phi$. For all $i \in N, x^{*} \in[0, \bar{\theta}-\underline{\theta}], \Sigma \in \mathcal{H}$, there exists a positive number $M(\Sigma)$ such that

$$
\Lambda_{i}\left(\Sigma \mid c_{-i}, s_{i}, \nu\right) \geq \Lambda_{i}\left(\Sigma \mid c_{-i}+x, s_{i}+x^{*}, \nu\right)-M(\Sigma)\left(\varepsilon_{0}(\nu)+\varepsilon_{x^{*}}(\nu)\right)
$$

for all $s_{i} \in[\underline{\theta}, \bar{\theta}], x \in[0, \bar{\theta}-\underline{\theta}]^{\left(\sum_{j \neq i} m_{j}-N+1\right)}$ such that $x \leq x^{*} \mathbf{1}$, and all $c_{-i} \in \mathcal{C}_{-i}$.

Proof: I prove the result by induction. First, I show it is true for $\Lambda_{i}\left(\bar{\Sigma} \mid c_{-i}, s_{i}, \nu\right)$. Notice there is only one way to obtain $\bar{\Sigma}$ and so:

$$
\operatorname{Prob}\left(\Sigma=\bar{\Sigma} \mid c_{-i}, s_{i}, \nu\right)=\Omega_{i}\left(\left(s_{j}>c_{m_{j}-1}\right)_{j \neq i} \mid s_{i}, \nu\right)
$$


In (10), set $x^{*}$ to zero and replace $\prod_{j \in J} F_{j}\left(\left(c_{j}-\theta\right) / \nu\right)$ with $\prod_{j \in J}\left(1-F_{j}\left(\left(c_{j}-\theta\right) / \nu\right)\right)$ to get $\operatorname{Prob}\left(\Sigma=\bar{\Sigma} \mid\right.$.), then Lemma 1 shows that the distance between $\Omega_{i}\left(\left(s_{j}>c_{m_{j}-1}\right)_{j \neq i} \mid s_{i}, \nu\right)$ and

$$
\int_{-\infty}^{\infty} \frac{1}{\nu} \prod_{j \neq i}\left(1-F_{j}\left(\frac{c_{m_{j}-1}-\theta}{\nu}\right)\right) f_{i}\left(\frac{s_{i}-\theta}{\nu}\right) d \theta
$$

is bounded by $\varepsilon_{0}(\nu) \rightarrow 0$ for all $s_{i} \in[\underline{\theta}, \bar{\theta}]$ and $c_{-i} \in \mathcal{C}_{-i}$. A simple change of variable $\left(\theta^{\prime}=\theta-x^{*}\right)$ would establish that (13) is equal to

$$
\int_{-\infty}^{\infty} \frac{1}{\nu} \prod_{j \neq i}\left(1-F_{j}\left(\frac{c_{m_{j}-1}+x^{*}-\theta}{\nu}\right)\right) f_{i}\left(\frac{s_{i}+x^{*}-\theta}{\nu}\right) d \theta
$$

Clearly, the distance between $\Omega_{i}\left(s_{-i}>\left(c_{m_{j}-1}\right)_{j \neq i}+x^{*} 1 \mid s_{i}+x^{*}, \nu\right)$ and (14) is bounded by $\varepsilon_{x^{*}}(\nu) \rightarrow 0$ for all $s_{i} \in[\underline{\theta}, \bar{\theta}]$ and $c_{-i} \in \mathcal{C}_{-i}$ by Lemma 1 . Since $\Omega_{i}\left(s_{-i}>\left(c_{m_{j}-1}\right)_{j \neq i}+\right.$ $\left.x^{*} \mathbf{1} \mid s_{i}+x^{*}, \nu\right) \leq \Omega_{i}\left(s_{-i}>\left(c_{m_{j}-1}\right)_{j \neq i}+x \mid s_{i}+x^{*}, \nu\right)$, then $\operatorname{Prob}\left(\Sigma=\bar{\Sigma} \mid c_{-i}, s_{i}, \nu\right) \leq$ $\operatorname{Prob}\left(\Sigma=\bar{\Sigma} \mid c_{-i}+x, s_{i}+x^{*}, \nu\right)+\varepsilon_{0}(\nu)+\varepsilon_{x^{*}}(\nu)$ for all $s_{i}, c_{-i} \in \mathcal{C}_{-i}$ and $x \in[0, \bar{\theta}-$ $\underline{\theta}]^{\left(\sum_{j \neq i} m_{j}-N+1\right)}$ such that $x \leq x^{*} \mathbf{1}$. Since $\Lambda_{i}\left(\bar{\Sigma} \mid c_{-i}, s_{i}, \nu\right)=1-\operatorname{Prob}\left(\Sigma=\bar{\Sigma} \mid c_{-i}, s_{i}, \nu\right)$, this shows the result for the greatest sum.

Now, suppose the claim is true for all sums greater or equal to $\Sigma^{\prime} \in \mathcal{H}$. Let $\mathrm{p}\left(\Sigma^{\prime}\right)=$ $\max \left\{\Sigma \in \mathcal{H}: \Sigma \prec \Sigma^{\prime}\right\}$ and show $\Lambda_{i}\left(\mathrm{p}\left(\Sigma^{\prime}\right) \mid c_{-i}, s_{i}, \nu\right)$ satisfies translation increasingness in the limit. There are now possibly several (but finitely many) ways to obtain $\mathrm{p}\left(\Sigma^{\prime}\right)$. Let $c_{-i}^{k}$ be the $k$-th combination of cutoffs of $i$ 's opponents that leads to $\mathrm{p}\left(\Sigma^{\prime}\right)$, and recall $\Lambda_{i}\left(\mathrm{p}\left(\Sigma^{\prime}\right) \mid c_{-i}, s_{i}, \nu\right)=1-\left(\operatorname{Prob}\left(\Sigma \geq \Sigma^{\prime} \mid c_{-i}, s_{i}, \nu\right)+\operatorname{Prob}\left(\Sigma=\mathrm{p}\left(\Sigma^{\prime}\right) \mid c_{-i}, s_{i}, \nu\right)\right.$. Note

$$
\operatorname{Prob}\left(\Sigma=\mathrm{p}\left(\Sigma^{\prime}\right) \mid c_{-i}, s_{i}, \nu\right)=\sum_{k} \Omega_{i}\left(\left(c_{k_{j}}^{k}<s_{j}<c_{k_{j}+1}^{k}\right)_{j \neq i} \mid s_{i}, \nu\right)
$$

for some $k_{j}$ and since there are only finitely many combinations of cutoffs that result in $\mathrm{p}\left(\Sigma^{\prime}\right)$, Lemma 1 implies the distance between $\operatorname{Prob}\left(\Sigma=\mathrm{p}\left(\Sigma^{\prime}\right) \mid c_{-i}, s_{i}, \nu\right)$ and

$$
\sum_{k} \int_{-\infty}^{\infty} \frac{1}{\nu} \prod_{j \neq i}\left(F_{j}\left(\frac{c_{k_{j}+1}^{k}-\theta}{\nu}\right)-F_{j}\left(\frac{c_{k_{j}}^{k}-\theta}{\nu}\right)\right) f_{i}\left(\frac{s_{i}-\theta}{\nu}\right) d \theta
$$

is bounded by $m\left(\mathrm{p}\left(\Sigma^{\prime}\right)\right) \varepsilon_{0}(\nu)$ for all $s_{i} \in[\underline{\theta}, \bar{\theta}]$ and $c_{-i} \in \mathcal{C}_{-i} \cdot{ }^{35}$ A simple change of variables $\left(\theta^{\prime}=\theta-x^{*}\right)$ would establish that (15) is equal to

$$
\sum_{k} \int_{-\infty}^{\infty} \frac{1}{\nu} \prod_{j \neq i}\left(F_{j}\left(\frac{c_{k_{j}+1}^{k}+x^{*}-\theta}{\nu}\right)-F_{j}\left(\frac{c_{k_{j}}^{k}+x^{*}-\theta}{\nu}\right)\right) f_{i}\left(\frac{s_{i}+x^{*}-\theta}{\nu}\right) d \theta
$$

Since $\operatorname{Prob}\left(\Sigma \geq \Sigma^{\prime} \mid c_{-i}, s_{i}, \nu\right)=1-\Lambda_{i}\left(\Sigma^{\prime} \mid c_{-i}, s_{i}, \nu\right)$, the above argument and the induction hypothesis imply that the distance between the sum of $\operatorname{Prob}\left(\Sigma \geq \Sigma^{\prime} \mid c_{-i}, s_{i}, \nu\right)$ and $\operatorname{Prob}\left(\Sigma=\mathrm{p}\left(\Sigma^{\prime}\right) \mid c_{-i}, s_{i}, \nu\right)$, and

$$
\operatorname{Prob}\left(\Sigma \geq \Sigma^{\prime} \mid c_{-i}+x^{*}, s_{i}+x^{*}, \nu\right)+\operatorname{Prob}\left(\Sigma=\mathrm{p}\left(\Sigma^{\prime}\right) \mid c_{-i}+x^{*}, s_{i}+x^{*}, \nu\right)
$$

\footnotetext{
${ }^{35}$ The distance is bounded by $\sum_{k} \varepsilon_{0}(\nu)$ where the summation counter only depends on $\mathrm{p}\left(\Sigma^{\prime}\right)$ and so there exists some $m\left(\mathrm{p}\left(\Sigma^{\prime}\right)\right)$.
} 
is less than $\left(M\left(\Sigma^{\prime}\right)+m\left(\mathrm{p}\left(\Sigma^{\prime}\right)\right)\right)\left(\varepsilon_{0}(\nu)+\varepsilon_{x^{*}}(\nu)\right)$ and this, for all $s_{i}$, and $c_{-i}$. Let $M\left(\mathrm{p}\left(\Sigma^{\prime}\right)\right)=$ $M\left(\Sigma^{\prime}\right)+m\left(\mathrm{p}\left(\Sigma^{\prime}\right)\right)$. But (17) is less than

$$
\operatorname{Prob}\left(\Sigma \geq \Sigma^{\prime} \mid c_{-i}+x, s_{i}+x^{*}, \nu\right)+\operatorname{Prob}\left(\Sigma=\mathrm{p}\left(\Sigma^{\prime}\right) \mid c_{-i}+x, s_{i}+x^{*}, \nu\right)
$$

for all $s_{i}, c_{-i}$ and $x \in[0, \bar{\theta}-\underline{\theta}]^{\left(\sum_{j \neq i} m_{j}-N+1\right)}$ such that $x \leq x^{*} \mathbf{1}$, which follows by definition of $\operatorname{Prob}\left(\Sigma \geq \mathrm{p}\left(\Sigma^{\prime}\right) \mid\right.$.). Indeed, $\Sigma$ is increasing in the players' actions and, when player $i$ 's opponents lower all their cutoffs player $i$ believes higher actions are more likely and so are greater sums.

Consequently,

$\operatorname{Prob}\left(\Sigma \geq \Sigma^{\prime} \mid c_{-i}, s_{i}, \nu\right)+\operatorname{Prob}\left(\Sigma=\mathrm{p}\left(\Sigma^{\prime}\right) \mid c_{-i}, s_{i}, \nu\right) \leq \operatorname{Prob}\left(\Sigma \geq \Sigma^{\prime} \mid c_{-i}+x, s_{i}+x^{*}, \nu\right)+$ $\operatorname{Prob}\left(\Sigma=\mathrm{p}\left(\Sigma^{\prime}\right) \mid c_{-i}+x, s_{i}+x^{*}, \nu\right)+M\left(\mathrm{p}\left(\Sigma^{\prime}\right)\right)\left(\varepsilon_{0}(\nu)+\varepsilon_{x^{*}}(\nu)\right)$,

for all $s_{i}, c_{-i}$ and $x$ which proves the claim for $\mathrm{p}\left(\Sigma^{\prime}\right)$. Since there are only a finite number of such sums, we proceed inductively and we are done.

Q.E.D

Corollary 4 Let $K=\left[\underline{\theta}-\nu e^{*}, 2 \bar{\theta}-\underline{\theta}+\nu e^{*}\right]$ be contained in the interior of the support of $\phi$. Letting $M(\Delta)$ be some positive number, then for all $i \in N, x^{*} \in[0, \bar{\theta}-\underline{\theta}], \Delta \in \mathcal{H}$,

$$
\Lambda_{i}\left(\Delta \mid c_{-i}, s_{i}, \nu\right) \geq \Lambda_{i}\left(\Delta \mid c_{-i}+x, s_{i}+x^{*}, \nu\right)-M(\Delta)\left(\varepsilon_{0}(\nu)+\varepsilon_{x^{*}}(\nu)\right)
$$

for all $s_{i} \in[\underline{\theta}, \bar{\theta}], x \in[0, \bar{\theta}-\underline{\theta}]^{\left(\sum_{j \neq i} m_{j}-N+1\right)}$ such that $x \leq x^{*} \mathbf{1}$, and all $c_{-i} \in \mathcal{C}_{-i}$.

Proof: Pick $c \in \cap_{j \neq i} A_{j}$ and notice $\bar{\Delta}=0$. Let $\zeta_{j}(c) \in A_{j}$ be the smallest action in $A_{j}$ such that $\zeta_{j}(c) \geq c$. Let $c_{j}^{*}$ denote $j$ 's cutoff point from which $j$ 's action is greater or equal to $\zeta_{j}(c)$. Then,

$$
\operatorname{Prob}\left(\Delta=0 \mid c_{-i}, s_{i}, \nu\right)=\Omega_{i}\left(\left(s_{j}>c_{j}^{*}\right)_{j \neq i} \mid s_{i}, \nu\right)
$$

As in the proof of Lemma 1, the claim holds for the greatest proportion. The rest of the proof then goes similarly.

Q.E.D

Corollary 5 In the private values case with uniform prior, beliefs $\Lambda_{i}\left(\Sigma \mid c_{-i}, s_{i}, \nu\right)$ (or $\left.\Lambda_{i}\left(\Delta \mid c_{-i}, s_{i}, \nu\right)\right)$ are translation increasing for every $\nu>0$.

Proof: The result follows immediately from Lemma 1 and Lemma 2 (or Corollary 4), and $\Psi_{i}^{\prime}\left(\theta \mid s_{i}, \nu\right)=(1 / \nu) f_{i}\left(\left(s_{i}-\theta\right) / \nu\right)$.

Q.E.D

Proof of Theorem 2: First, I prove $\cup_{\nu>0} \mathcal{C}_{\nu}=\mathcal{C}^{2}$. Trivially $(c, c) \in \cup_{\nu>0} \mathcal{C}_{\nu}$ for all $c \in \mathcal{C}$. Now, take any $\left(c, c^{\prime}\right) \in \mathcal{C}^{2}$ with $c \neq c^{\prime}$. Let $l\left(c_{-i}, s_{i}, \nu\right)$ and $r\left(s_{i}\right)$ be respectively the left-hand side and right-hand side of (8). Say $\left(c_{-i}, c_{\beta, \alpha}^{r}(\nu)\right)$ satisfies (8) at $\nu$ : $l\left(c_{-i}, c_{\beta, \alpha}^{r}(\nu), \nu\right)=r\left(c_{\beta, \alpha}^{r}(\nu)\right)$. Let $x_{j, k_{j}}=\left|c_{j, k_{j}}^{\prime}-c_{j, k_{j}}\right|, x=\left(x_{j, k_{j}}\right)_{j \neq i}$ and 
$x^{*}=\max _{j \neq i} \max _{k_{j}} x_{j, k_{j}}$. By means of contradiction, suppose $c_{\beta, \alpha}^{\prime r}(\nu)=c_{\beta, \alpha}^{r}(\nu)+x^{*}$. By definition, $\left(c_{-i}^{\prime}, c_{\beta, \alpha}^{\prime r}(\nu)\right)$ must also satisfy (8) at $\nu$, and so by Lemma $2:{ }^{36}$

$$
\sum_{\{\Sigma: \bar{\Sigma}>\Sigma \geq \underline{\Sigma}\}}\left(\Lambda_{i}\left(\rho(\Sigma) \mid c_{-i}, c_{\beta, \alpha}^{r}(\nu), \nu\right)+\mu(\nu)\right) \delta\left(\Sigma, \rho(\Sigma), c_{\beta, \alpha}^{\prime r}(\nu)\right) \geq r\left(c_{\beta, \alpha}^{\prime r}(\nu)\right)
$$

where $\mu(\nu) \rightarrow 0$ as $\nu \rightarrow 0$. Since $m_{j}<\infty$ and $\pi_{i}$ is bounded as a continuous function on a compact set, then we can rewrite this last inequality as

$$
\sum \Lambda_{i}\left(\rho(\Sigma) \mid c_{-i}, c_{\beta, \alpha}^{r}(\nu), \nu\right) \delta\left(\Sigma, \rho(\Sigma), c_{\beta, \alpha}^{\prime r}(\nu)\right)+\vartheta_{i}(\nu) \geq r\left(c_{\beta, \alpha}^{\prime r}(\nu)\right)
$$

where $\vartheta_{i}(\nu) \rightarrow 0$ as $\nu \rightarrow 0$. Since $r\left(s_{i}+x^{*}\right)-r\left(s_{i}\right)$ is strictly positive by (A3) and continuous in $s_{i}$, then it has a strictly positive minimum over $[\underline{\theta}, \bar{\theta}]$, call it $m^{*}$. Let $m^{* *}=m^{*} / 2$. Note since $l\left(c_{-i}, c_{\beta, \alpha}^{r}(\nu), \nu\right)=r\left(c_{\beta, \alpha}^{r}(\nu)\right)$, then

$$
\sum \Lambda_{i}\left(\rho(\Sigma) \mid c_{-i}, c_{\beta, \alpha}^{r}(\nu), \nu\right) \delta\left(\Sigma, \rho(\Sigma), c_{\beta, \alpha}^{\prime r}(\nu)\right)+m^{* *} \leq r\left(c_{\beta, \alpha}^{r}(\nu)\right)+m^{* *}<r\left(c_{\beta, \alpha}^{\prime r}(\nu)\right)
$$

Since $\vartheta_{i}(\nu) \rightarrow 0$, (19) and (20) lead to a contradiction as soon as $\vartheta_{i}(\nu)$ is less than $m^{* *}$. It means that there exists $\nu$ small enough such that $l\left(c_{-i}^{\prime}, c_{\beta, \alpha}^{\prime r}(\nu), \nu\right)<r\left(c_{\beta, \alpha}^{\prime r}(\nu)\right)$, hence the contradiction with $\left(c_{-i}^{\prime}, c_{\beta, \alpha}^{\prime r}(\nu)\right)$ verifying (8). Given this last inequality, it is also a contradiction for $x^{*}>\max _{j \neq i} \max _{k_{j}} x_{j, k_{j}}$ by (A2), (A3), (A4) and (A7). Therefore, the $\nu$ from which we reach a contradiction only depends on $i, \beta, \alpha$ and on the direction: from $\left(c_{-i}, c_{\beta, \alpha}^{r}\right)$ to $\left(c_{-i}^{\prime}, c_{\beta, \alpha}^{\prime r}\right)$, or the other way. Since there are only finitely many players, actions and directions, then there exists $\underline{\nu}>0$ such that for all $i \in N$, $\mathrm{t}\left(c_{i}^{\prime r}(\nu), c_{i}^{r}(\nu)\right)<d\left(c_{-i}^{\prime}, c_{i}\right)$ for all $\nu<\underline{\nu}$. The fictitious cutoffs generated by these real cutoffs then form a shrinking map. That is, $d\left(b r(\nu)(c), b r(\nu)\left(c^{\prime}\right)\right)<d\left(c, c^{\prime}\right)$ for all $\nu<\underline{\nu}$. Therefore, $\left(c, c^{\prime}\right) \in \cup_{\nu>0} \mathcal{C}_{\nu}$.

Secondly, I want to show that for any $\left(c, c^{\prime}\right) \in \mathcal{C}^{2}$, there exist $\underline{\nu}$ and a neighborhood $\mathcal{U}$ of $\left(c, c^{\prime}\right)$ in the product topology of $\mathcal{C}^{2}$ such that $\mathcal{U} \subset \mathcal{C}_{\underline{\nu}}$. To simplify notation, I do not indicate the dependance on $a_{\alpha}$ and $a_{\beta}$ because they live in a finite set and the minimum over all $\left\{a_{\alpha}, a_{\beta}\right\}$ of a continuous function is itself continuous.

Let us specify $\mu_{x^{*}}$ from the first part of the theorem and then construct $\vartheta_{i}$. Let $\mu_{x^{*}}(\nu)=$ $\max _{\Sigma} M(\Sigma)\left(\varepsilon_{0}(\nu)+\varepsilon_{x^{*}}(\nu)\right)$ where $x^{*}=\max _{j \neq i} \max _{k_{j}}\left|c_{k_{j}}^{\prime}-c_{k_{j}}\right|$. By the Maximum Theorem, $\rho_{x^{*}}(\nu)$ is continuous in $x^{* 37}$ and then, so are $\varepsilon_{x^{*}}(\nu)=\max \left\{1-1 / \rho_{x^{*}}(\nu), \rho_{x^{*}}(\nu)-1\right\}$ and $\mu_{x^{*}}(\nu)$ for each $\nu$. Moreover, notice the minimum of $r\left(s_{i}+x^{*}\right)-r\left(s_{i}\right)$ over $[\underline{\theta}, \bar{\theta}]$ only depends on $i$ and $x^{*}$ and thus its half, $m_{i}^{* *}\left(x^{*}\right)$, is continuous in $x^{*}$ by the Maximum Theorem. Now, let $b_{i}=\max _{s_{i} \in[\theta, \bar{\theta}]} \sum_{\Sigma} \delta\left(\Sigma, \rho(\Sigma), s_{i}\right)$, and define $\vartheta_{i}\left(\nu, x^{*}\right)=b_{i} \mu_{x^{*}}(\nu)$. Since $\mu_{x^{*}}(\nu) \rightarrow 0$, then for any $i \in N$, there exists $\underline{\nu}_{i}>0$ such that

$$
\vartheta_{i}\left(\underline{\nu}_{i}, x^{*}\right)<m_{i}^{* *}\left(x^{*}\right) .
$$

\footnotetext{
${ }^{36}$ For example, let $\mu(\nu)=\max _{\Sigma} M(\Sigma)\left(\varepsilon_{0}(\nu)+\varepsilon_{x^{*}}(\nu)\right)$. By Lemma 2, we know $\Lambda_{i}\left(\Sigma \mid c_{-i}, s_{i}, \nu\right) \geq$ $\Lambda_{i}\left(\Sigma \mid c_{-i}+x, s_{i}+x^{*}, \nu\right)-\mu(\nu) \geq \Lambda_{i}\left(\Sigma \mid c_{-i}^{\prime}, s_{i}+x^{*}, \nu\right)-\mu(\nu)$ and since $\delta($.$) is positive, then we get the$ inequality.

${ }^{37}$ See discussion preceding the proof of Lemma 1.
} 
As $x^{*}$ is continuous in $\left(c, c^{\prime}\right)$, then for any $i \in N$ there exists a neighborhood of $\left(c_{-i}, c_{-i}^{\prime}\right)$ such that $(21)$ is satisfied at $\underline{\nu}_{i}$. Therefore, there is a neighborhood $\mathcal{U}$ of $\left(c, c^{\prime}\right)$ and $\underline{\nu}>0$ such that $\mathcal{U} \subset C_{\underline{\nu}}$.

Q.E.D

Proof of Corollary 2: Suppose by way of contradiction that there is $\iota>0$ such that for all $\underline{\nu}>0$, there exists $\nu<\underline{\nu}$ for which $d\left(\underline{e}_{\nu}, \bar{e}_{\nu}\right)>\iota$. Since $\left\{\left(\underline{e}_{\nu}, \bar{e}_{\nu}\right)\right\} \subset \mathcal{C}^{2}$ and $\mathcal{C}^{2}$ is compact, then the sequence has at least one cluster point in $\mathcal{C}^{2}$. For such $\iota$ to exist, there must be a cluster point of the sequence, denoted $(\underline{e}, \bar{e})$, such that $\underline{e} \neq \bar{e}$. By Theorem 2 , there exists $\underline{\nu}$ such that a neighborhood $\mathcal{U}$ of $(\underline{e}, \bar{e})$ is a subset of $\mathcal{C}_{\underline{\nu}}$. Now, take two disjoint neighborhoods $\underline{\mathcal{U}}$ and $\overline{\mathcal{U}}$ in $\mathcal{C}$, respectively of $\underline{e}$ and $\bar{e}$, such that $\underline{\mathcal{U}} \times \overline{\mathcal{U}} \subset \mathcal{U}$. Notice such neighborhoods exist because $\mathcal{U}$ is an open set in the product topology. Since $(\underline{e}, \bar{e})$ is a cluster point, then there exists $\nu^{\prime}<\underline{\nu}$ such that $\left(\underline{e}_{\nu^{\prime}}, \bar{e}_{\nu^{\prime}}\right) \in \underline{\mathcal{U}} \times \overline{\mathcal{U}} \subset \mathcal{U} \subset \mathcal{C}_{\underline{\nu}}$ which is a contradiction.

Q.E.D

Proposition 6 In the common values case with uniform prior, beliefs $\Lambda_{i}\left(g, \theta \mid c_{-i}, s_{i}, \nu\right)$ are increasing in translation for every $\nu>0$.

Proof: Take any $c_{-i} \in \mathcal{C}_{-i}$ and let $c_{-i}^{k}$ be the $k$-th combination of cutoffs of $i$ 's opponents that leads to $g$ and recall there are only finitely many such combinations. Take $x \in$ $[0, \bar{\theta}-\underline{\theta}]^{\left(\sum_{j \neq i} m_{j}-N+1\right)}$ and $x^{*} \in[0, \bar{\theta}-\underline{\theta}]$ with $x^{*} \mathbf{1} \geq x$. From (5) and using the same change of variables as in Lemma 1 , we get that $\Lambda_{i}\left(g, \theta \mid c_{-i}+x^{*} \mathbf{1}, s_{i}+x^{*}, \nu\right)$ is equal to

$$
\sum_{k} \int_{\frac{s_{i}+x^{*}-\theta}{\nu}}^{\infty} \prod_{j \in J}\left(F_{j}\left(\frac{c_{k_{j}+1}^{k}-s_{i}+\nu z}{\nu}\right)-F_{j}\left(\frac{c_{k_{j}}^{k}-s_{i}+\nu z}{\nu}\right)\right) f(z) d z .
$$

and $\Lambda_{i}\left(g, \theta \mid c_{-i}, s_{i}, \nu\right)$ is equal to

$$
\sum_{k} \int_{\frac{s_{i}-\theta}{\nu}}^{\infty} \prod_{j \in J}\left(F_{j}\left(\frac{c_{k_{j}+1}^{k}-s_{i}+\nu z^{\prime}}{\nu}\right)-F_{j}\left(\frac{c_{k_{j}}^{k}-s_{i}+\nu z^{\prime}}{\nu}\right)\right) f\left(z^{\prime}\right) d z^{\prime} .
$$

Since $f_{i}$ is positive-valued and $c_{k_{j}+1}^{k} \geq c_{k_{j}}^{k}$, (23) is bigger than (22). I have already argued that $\Lambda_{i}\left(g, \theta \mid c_{-i}+x, s_{i}+x^{*}, \nu\right) \leq \Lambda_{i}\left(g, \theta \mid c_{-i}+x^{*}, s_{i}+x^{*}, \nu\right)$ which leads to

$$
\Lambda_{i}\left(g, \theta \mid c_{-i}+x, s_{i}+x^{*}, \nu\right) \leq \Lambda_{i}\left(g, \theta \mid c_{-i}, s_{i}, \nu\right) .
$$

I am done as $\theta$ was arbitrary.

Unlike the private values case where $\theta=\infty,(23)$ and (22) are not equal. Under a uniform prior (or in the limit), only the difference between her signal and the others' matters to player $i$ to formulate her beliefs about the opposing profile of actions, and so she thinks each value of the aggregate is as probable when she receives $s_{i}+x^{*}$ and the others play $c_{-i}+x^{*} \mathbf{1}$ as when she receives $s_{i}$ and the others play $c_{-i}$. However, she expects now higher $\theta$ in the first situation which she did not under private values and this explains why (23) is bigger than (22). 
At this point, it should be clear that every translation increasingness type of properties that is satisfied for every $\nu$ by the beliefs under a uniform prior will also be satisfied uniformly in the limit by the corresponding beliefs under a general prior assumption. Indeed, for a general prior, $\Lambda_{i}\left(g, \theta \mid c_{-i}, s_{i}, \nu\right)$ and $\Lambda_{i}\left(g, \theta \mid c_{-i}+x^{*}, s_{i}+x^{*}, \nu\right)$ become arbitrarily close to (22) and (23) uniformly in $s_{i}, c_{-i}, \theta$. This comes from a similar result to Lemma 1 for $\Omega\left(c_{-i}, \theta \mid s_{i}, \nu\right)$. Consequently, I omit the proof of the next result.

Lemma 3 Let $K=\left[\underline{\theta}-\eta-\nu e^{*}, 2 \bar{\theta}-\underline{\theta}+3 \eta+\nu e^{*}\right]$ be contained in the interior of the support of $\phi$. Letting $M(\Sigma)$ be some positive number, then for all $i \in N, x^{*} \in[0, \bar{\theta}-\underline{\theta}+2 \eta]$, $\Sigma \in \mathcal{H}$

$$
\Lambda_{i}\left(\Sigma, \theta \mid c_{-i}, s_{i}, \nu\right) \geq \Lambda_{i}\left(\Sigma, \theta \mid c_{-i}+x, s_{i}+x^{*}, \nu\right)-M(\Sigma)\left(\varepsilon_{0}(\nu)+\varepsilon_{x^{*}}(\nu)\right)
$$

for all $s_{i} \in[\underline{\theta}-\eta, \bar{\theta}+\eta], x \in[0, \bar{\theta}-\underline{\theta}+2 \eta]^{\left(\sum_{j \neq i} m_{j}-N+1\right)}$ such that $x \leq x^{*} \mathbf{1}, \theta \in \mathbb{R}$ and all $c_{-i} \in \mathcal{C}_{-i}$.

The next lemma is a technical result that will be useful in proving Theorem $4 .{ }^{38}$

Lemma 4 Suppose state monotonicity is decreasing. Then there exists a positive function $\delta^{*}\left(\Sigma^{*}, \Sigma, \theta\right)$ from $\mathcal{H}^{2} \times \mathbb{R}$ to $\mathbb{R}_{+}$that is decreasing in $\left(\Sigma^{*}, \theta\right)$ and such that for all $\Sigma, s_{i}, c_{-i}$ and $\nu$ :

$$
\int_{\mathcal{H} \times \mathbb{R}} \delta^{*}\left(\Sigma^{*}, \Sigma, \theta\right) d \Lambda_{i}\left(\Sigma^{*}, \theta \mid c_{-i}, s_{i}, \nu\right)=\int_{\theta \in \mathbb{R}} \delta(\Sigma, \rho(\Sigma), \theta) \Lambda_{i}\left(\rho(\Sigma), d \theta \mid c_{-i}, s_{i}, \nu\right)
$$

Proof: Define

$$
\delta^{*}\left(\Sigma^{*}, \Sigma, \theta\right)= \begin{cases}\delta(\Sigma, \rho(\Sigma), \theta) & \text { if } \Sigma^{*}<\rho(\Sigma) \\ 0 & \text { otherwise }\end{cases}
$$

and notice $\delta^{*}$ is decreasing in $\left(\Sigma^{*}, \theta\right)$ by construction, $(D S M)$ and supermodularity. It is also positive by supermodularity. The proof then follows by Fubini's theorem. Q.E.D

Proof of Theorem 3: Recall that for $\nu<\eta / e^{*}$, all real cutoffs exist. Suppose that state monotonicity is decreasing. By strict first-order stochastic dominance of $\Psi_{i}$ and Lemma 4, Equation 24, which is the analog of Equation 8 but in the common values, has a unique solution. ${ }^{39}$ From then, the proof is similar to the proof of Proposition 4 . All the real cutoffs shrink as functions of the opposing profile which translates to the fictitious cutoffs by Theorem 1. Uniqueness then follows from the contraction of $b r(\nu)$. Q.E.D

Proof of Theorem 4: First, I prove that $\cup_{\nu>0} \mathcal{C}_{\nu}=\mathcal{C}^{2}$. Trivially, $(c, c) \in \cup_{\nu>0} \mathcal{C}_{\nu}$ for all $c \in \mathcal{C}$. Now, take any $\left(c, c^{\prime}\right) \in \mathcal{C}^{2}$ with $c \neq c^{\prime}$. From Equation 3, we can write $i$ 's utility to playing $a_{i}$ when $j$ plays according to $c_{j}$ :

$E \pi_{i}\left(a_{i}, c_{-i}, s_{i}\right)=\int_{\theta \in \mathbb{R}} \pi_{i}\left(a_{i}, \underline{\Sigma}, \theta\right) \Lambda_{i}\left(\rho(\underline{\Sigma}), d \theta \mid c_{-i}, s_{i}, \nu\right)+\int_{\theta \in \mathbb{R}} \sum_{\{\Sigma: \bar{\Sigma}>\Sigma>\underline{\Sigma}\}} \pi_{i}\left(a_{i}, \Sigma, \theta\right)\left(\Lambda_{i}(\rho(\Sigma)\right.$

\footnotetext{
${ }^{38}$ I thank Federico Echenique for suggesting this Lemma.

${ }^{39}$ See the proof of Theorem 4.
} 


$$
\left.\left., d \theta \mid c_{-i}, s_{i}, \nu\right)-\Lambda_{i}\left(\Sigma, d \theta \mid c_{-i}, s_{i}, \nu\right)\right)+\int_{\theta \in \mathbb{R}} \pi_{i}\left(a_{i}, \bar{\Sigma}, \theta\right)\left(d \Psi_{i}\left(\theta \mid s_{i}, \nu\right)-\Lambda_{i}\left(\bar{\Sigma}, d \theta \mid c_{-i}, s_{i}, \nu\right)\right)
$$

which can be rewritten,

$$
\begin{aligned}
E \pi_{i}\left(a_{i}, c_{-i}, s_{i}\right) & =\int_{\theta \in \mathbb{R}} \pi_{i}\left(a_{i}, \bar{\Sigma}, \theta\right) d \Psi_{i}\left(\theta \mid s_{i}, \nu\right)+\int_{\theta \in \mathbb{R}} \sum\left(\pi_{i}\left(a_{i}, \Sigma, \theta\right)-\pi_{i}\left(a_{i}, \rho(\Sigma), \theta\right)\right) \Lambda_{i}(\rho \\
\left.(\Sigma), d \theta \mid s_{i}, \nu\right) . &
\end{aligned}
$$

Now, pick $\beta, \alpha \in\left\{1, \ldots, m_{i}\right\}$ such that $\beta>\alpha$. By definition, $c_{\beta, \alpha}^{r}(\nu)$ is the signal $s_{i}$ that verifies $E d \pi_{i}\left(a_{i, \beta}, a_{i, \alpha}, c_{-i}, s_{i}, \nu\right)=0$ which by the last equation is equivalent to searching for the unique $s_{i}$ such that ${ }^{40}$

$$
\left.\sum \int_{\theta \in \mathbb{R}} \delta(\Sigma, \rho(\Sigma), \theta) \Lambda_{i}\left(\rho(\Sigma), d \theta \mid c_{-i}, s_{i}, \nu\right)\right)=\int_{\theta \in \mathbb{R}}\left(d \pi_{i}\left(a_{\beta}, a_{\alpha}, \bar{\Sigma}, \theta\right)\right) d \Psi_{i}\left(\theta \mid s_{i}, \nu\right)
$$

Let $l\left(c_{-i}, s_{i}, \nu\right)$ and $r\left(s_{i}, \nu\right)$ be respectively the left-hand side and right-hand side of (24). Let $x_{j, k_{j}}=\left|c_{j, k_{j}}^{\prime}-c_{j, k_{j}}\right|, x=\left(x_{j, k_{j}}\right)_{j \neq i}$ and $x^{*}=\max _{j \neq i} \max _{k_{j}} x_{j, k_{j}}$. By way of contradiction, suppose $c_{\beta, \alpha}^{\prime r}(\nu)=c_{\beta, \alpha}^{r}(\nu)+x^{*}$. By definition, $\left(c_{-i}^{\prime}, c_{\beta, \alpha}^{\prime r}(\nu)\right)$ must also satisfy (24) at $\nu$. By strict first-order stochastic dominance, $r\left(s_{i}, \nu\right)$ is strictly increasing in $s_{i}$. Denote $\int \pi_{i}\left(a_{i}, \Sigma, \theta\right) d \Psi_{i}\left(\theta \mid s_{i}, \nu\right)$ by $u\left(a_{i}, \Sigma, s_{i}\right)$. Since $\Psi_{i}\left(\theta \mid s_{i}, \nu\right)$ is continuous in $s_{i} \in[\underline{\theta}-\eta, \bar{\theta}+\eta]$, $u_{i}\left(a_{i}, \Sigma, s_{i}\right)$ is continuous in $s_{i}$. By (A3), $r\left(s_{i}+x^{*}, \nu\right)-r\left(s_{i}, \nu\right)$ has then a strictly positive minimum over $[\underline{\theta}, \bar{\theta}]$, call it $m^{*}\left(x^{*}, \nu\right)$, and $m^{*}\left(x^{*}, \nu\right) \rightarrow m^{*}\left(x^{*}\right)>0$. ${ }^{41}$ Now the proof goes similarly to Theorem 2. By Lemma 3, there is an upper bound as to how much $\Lambda_{i}\left(\rho(\Sigma), \theta \mid c_{-i}, c_{\beta, \alpha}^{r}(\nu), \nu\right)$ can stochastically dominate $\Lambda_{i}\left(\rho(\Sigma), \theta \mid c_{-i}^{\prime}, c_{\beta . \alpha}^{\prime r}(\nu), \nu\right)$, that is, $M(\Sigma)\left(\varepsilon_{0}(\nu)+\varepsilon_{x^{*}}(\nu)\right)$. Since it goes to zero as $\nu \rightarrow 0$, then by Lemma 4 there exists $\underline{\nu}$ below which we reach, $l\left(c_{-i}, c_{\beta, \alpha}^{\prime r}(\nu), \nu\right)<r\left(c_{\beta, \alpha}^{\prime r}(\nu), \nu\right)$, a contradiction. By (A2), (A3), (A4) and (A7), it is also a contradiction for $x^{*}>\max _{j \neq i} \max _{k_{j}}\left|c_{j, k_{j}}^{\prime}-c_{j, k_{j}}\right|$. The contraction carries over to the fictitious cutoffs and so to the best-response, which completes the first part of the proof.

Given $\varepsilon_{x^{*}}(\nu)$ and $m^{*}\left(x^{*}, \nu\right)$ (and $m^{*}\left(x^{*}\right)$ ) are continuous in $x^{*}$ for each $\nu$, the same argument as in Theorem 2 applies to establish the second part of the claim.

Q.E.D

\section{References}

[1] Aliprantis C., K. Border (1999), Infinite Dimensional Analysis, Springer, Berlin.

[2] Athey S. (2001), "Single Crossing Properties and the Existence of Pure Strategy Equilibria in Games of Incomplete Information," Econometrica, 69, 861-90.

[3] Carlsson H., E. van Damme (1993), "Global Games and Equilibrium Selection," Econometrica, 61, 989-1018.

\footnotetext{
${ }^{40}$ There is only one $s_{i}$ verifying this equation for each $\left(c_{-i}, \nu\right)$ and $\alpha, \beta$ by Lemma 4 and (strict) first-order stochastic dominance.

${ }^{41}$ The definition of $m^{*}\left(x^{*}\right)$ is in the proof of Theorem 2 .
} 
[4] Frankel D.M., S. Morris, A. Pauzner (2003), "Equilibrium Selection in Global Games with Strategic Complementarities," Journal of Economy Theory, 108, 1-44.

[5] Goldstein I., A. Pauzner (2005), "Demand Deposit Contracts and the Probability of Bank Runs," Journal of Finance, 60, 1293-1327.

[6] Harrison R. (2003), "Equilibrium Selection in Global Games with Strategic Subsitutes," Working Paper, Georgetown University.

[7] Harsanyi J.C. (1973), "Games with Randomly Disturbed Payoffs: A New Rationale for Mixed Strategy Equilibrium Points," International Journal of Game Theory, 2, 1-23.

[8] McAdams D. (2003), "Isotone Equilibrium in Games of Incomplete Information," Econometrica, 71, 1191-214.

[9] Mason R., Á. Valentinyi (2003), "Independence and Heterogeneity in Games of Incomplete Information," CEPR Discussion Paper 4177.

[10] Mathevet L. (2005), "Finite Global Games and Contraction," mimeo, Caltech.

[11] Milgrom P., J. Roberts (1990), "Rationalizability Learning, and Equilibrium in Games with Strategic Complementarities," Econometrica, 58, 1255-1277.

[12] Milgrom P., C. Shannon (1994), "Monotone Comparative Statics," Econometrica, 62, 157-180.

[13] Morris S., H.S. Shin (1998), "Unique Equilibrium in a Model of Self-Fulfilling Currency Attacks," American Economic Review, 88, 587-97.

[14] Morris S., H.S. Shin (2004), "Coordination Risk and the Price of Debt," European Economic Review, 48, 133-153.

[15] Morris S., H.S. Shin (2002), "Global Games: Theory and Applications," in Advances in Economics and Econometrics, the Eighth World Congress (edited by M. Dewatripont, L. Hansen and S. Turnovsky), Cambridge University Press.

[16] Morris S., H.S. Shin (2005), "Heterogeneity and Uniqueness in Interaction Games," in The Economy as an Evolving Complex System III (edited by L. Blume and S. Durlauf. Santa Fe Institute Studies in the Sciences of Complexity), New York: Oxford University Press.

[17] Nadler S. B. Jr (1968), "Sequences of Contractions and Fixed Points," Pacific Journal of Mathematics, $27,579-585$.

[18] Oury M. (2005), "Multidimensional Global Games," mimeo, HEC School of Management.

[19] Shaked M., J.G. Shanthikumar (1994), Stochastic Orders and their Applications, Boston: Academic Press.

[20] Takahashi S. (2004), "The Number of Pure Nash Equilibria in a Random Game with Nondecreasing Best Responses," Working Paper, Harvard University.

[21] Topkis D.M. (1998), Supermodularity and Complementarity, Princeton, New Jersey.

[22] Toxvaerd F. (2003), "Strategic Merger Waves: A Theory of Musical Chairs," Center for Rationality and Interactive Decision Theory, Hebrew University, Jerusalem, Discussion Paper 359.

[23] Vives X. (1990), "Nash Equilibrium with Strategic Complementarities," Journal of Mathematical Economics, 19, 305-321.

[24] Van Zandt T., X. Vives (2003), "Monotone Equilibria in Bayesian Games of Strategic Complementarities," CEPR Discussion Paper 4103. 
[25] Vives X. (2004), "Complementarities and Games: New Developments," CEPR Discussion Paper 4742.

[26] Zimper A. (2004), "Dominance-Solvable Lattice Games," Sonderforschungsbereich 504 Publications, 04-18. 